\title{
Threshold Dynamics in Stochastic SIRS Epidemic Models with Nonlinear Incidence and Vaccination
}

\author{
Lei Wang, ${ }^{1}$ Zhidong Teng, ${ }^{2}$ Tingting Tang, ${ }^{2}$ and Zhiming $\mathrm{Li}^{2}$ \\ ${ }^{1}$ Department of Medical Engineering and Technology, Xinjiang Medical University, Urumqi 830011, China \\ ${ }^{2}$ College of Mathematics and System Sciences, Xinjiang University, Urumqi 830046, China
}

Correspondence should be addressed to Zhidong Teng; zhidong_teng@sina.com

Received 30 July 2016; Accepted 21 November 2016; Published 16 January 2017

Academic Editor: Giuseppe Pontrelli

Copyright (C) 2017 Lei Wang et al. This is an open access article distributed under the Creative Commons Attribution License, which permits unrestricted use, distribution, and reproduction in any medium, provided the original work is properly cited.

In this paper, the dynamical behaviors for a stochastic SIRS epidemic model with nonlinear incidence and vaccination are investigated. In the models, the disease transmission coefficient and the removal rates are all affected by noise. Some new basic properties of the models are found. Applying these properties, we establish a series of new threshold conditions on the stochastically exponential extinction, stochastic persistence, and permanence in the mean of the disease with probability one for the models. Furthermore, we obtain a sufficient condition on the existence of unique stationary distribution for the model. Finally, a series of numerical examples are introduced to illustrate our main theoretical results and some conjectures are further proposed.

\section{Introduction}

As is well known, transmissions of many infectious diseases are inevitably affected by environment white noise, which is an important component in realism, because it can provide some additional degrees of realism compared to their deterministic counterparts. Therefore, in recent years, stochastic differential equation (SDE) has been used widely by many researchers to model the dynamics of spread of infectious disease (see [1-5] and the references cited therein). There are different possible approaches to include effects in the model. Here, we mainly introduce three approaches. The first one is through time Markov chain model to consider environment noise in SIS model (see, e.g., [6] and the references cited therein). The second is with parameters perturbation (see $[2,5,7]$ and the references cited therein). The last issue to model stochastic epidemic system is to perturb around the positive equilibria of deterministic models (see, e.g., $[1,8,9]$ and the references cited therein).

Now, we consider stochastic epidemic models with parameters perturbation. The incidence rate of a disease denotes the number of new cases per unit time, and this plays an important role in the study of mathematical epidemiology. In many epidemic models, the bilinear incidence rate $\beta S I$ is frequently used (see $[2,5,7,8,10-17])$, and the saturated incidence rate $\beta S I /(1+a I)$ is also frequently used (see, e.g., [18-22]). Comparing with bilinear incidence rate and saturated incidence rate, Lahrouz and Omari [23] and Liu and Chen [24] introduced a nonlinear incidence rate $\beta S I / \varphi(I)$ into stochastic SIRS epidemic models. In [25], Tang et al. investigated a class of stochastic SIRS epidemic models with nonlinear incidence rate $\beta f(S) g(I)$ :

$$
\begin{aligned}
d S= & (\Lambda-\beta f(S) g(I)-\mu S+\delta R) d t \\
& -\sigma f(S) g(I) d B(t), \\
d I= & (\beta f(S) g(I)-(\mu+\alpha+\gamma) I) d t \\
& +\sigma f(S) g(I) d B(t), \\
d R= & (\gamma I-(\mu+\delta) R) d t .
\end{aligned}
$$

Lahrouz et al. [26] studied a deterministic SIRS epidemic model with nonlinear incidence rate $\beta S I / \varphi(I)$ and vaccination. If the transmission of the disease is changed by nonlinear incidence rate $\beta f(S) g(I)$, and to make the model more realistic, let us suppose that the death rates of the three classes in the population are different, then a 
more general deterministic SIRS model is described by the following ordinary differential equation:

$$
\begin{aligned}
& \dot{S}=(1-q) \Lambda-\beta f(S) g(I)-\left(d_{S}+p\right) S+\varepsilon R, \\
& \dot{I}=\beta f(S) g(I)-\left(d_{I}+\gamma\right) I, \\
& \dot{R}=q \Lambda+p S+\gamma I-\left(d_{R}+\varepsilon\right) R,
\end{aligned}
$$

where $S(t), I(t)$, and $R(t)$ denote the numbers of susceptible, infectious, and recovered individuals at time $t$, respectively. $\Lambda$ denote a constant input of new members into the susceptible per unit time. $q$ is the rate of vaccination for the new members. $p$ is the rate of vaccination for the susceptible individuals. $d_{S}$ is the natural mortality rate or the removal rate of the $S . d_{I}$ is the removal rate of the infectious and usually is the sum of natural mortality rate and disease-induced mortality rate. $d_{R}$ is the removal rate of the recovered individual. $\gamma$ is the recovery rate of infective individual. $\varepsilon$ is the rate at which the recovered individual loses immunity. $\beta$ represents the transmission coefficient between compartments $S$ and $I$, and $\beta f(S) g(I)$ denotes the incidence rate of the disease. For biological reasons, we usually assume that functions $f(S)$ and $g(I)$ satisfy the following properties:

$\left(\mathbf{H}_{\mathbf{l}}\right) g(I)$ is two-order continuously differentiable function; $g(I) / I$ is monotonically nondecreasing with respect to $I ; g(0)=0$ and $g^{\prime}(0)>0$.

$\left(\mathbf{H}_{2}\right) f(S)$ is two-order continuously differentiable function; $f^{\prime}(S) \geq 0$ and $f^{\prime \prime}(S) \leq 0$ for all $S \geq 0$, and $f(0)=0$.

It is well known that the basic reproduction number for model (2) is defined by $R_{0}=\beta f\left(S_{0}\right) g^{\prime}(0) /\left(d_{I}+\gamma\right)$, where $S_{0}=$ $\left[(1-q) d_{R}+\varepsilon\right] \Lambda /\left(d_{S}\left(d_{R}+\varepsilon\right)+p d_{R}\right)$. Applying the Lyapunov function method and the theory of persistence for dynamical systems, we can prove that, when $R_{0}<1$, model (2) has a globally asymptotically stable disease-free equilibrium $E_{0}=$ $\left(S_{0}, 0, R_{0}\right)$ and, when $R_{0}>1$, model (2) has a unique endemic equilibrium $E^{*}\left(S^{*}, I^{*}, R^{*}\right)$ and disease $I$ is permanent.

In this paper, we extend model (1) to more general cases. As in [11], taking into account the effect of randomly fluctuating environment, we assume that fluctuations in the environment will manifest themselves mainly as fluctuations in parameters $\beta, d_{S}, d_{I}$, and $d_{R}$ in model (2) change to random variables $\widetilde{\beta}, \tilde{d}_{S}, \tilde{d}_{I}$, and $\tilde{d}_{R}$ such that

$$
\begin{gathered}
\tilde{\beta}=\beta+\text { error }_{0}, \\
\tilde{d}_{S}=d_{S}+\text { error }_{1}, \\
\tilde{d}_{I}=d_{I}+\text { error }_{2}, \\
\widetilde{d}_{R}=d_{R}+\text { error }_{3} .
\end{gathered}
$$

Accordingly, model (2) becomes

$$
\begin{aligned}
d S= & \left((1-q) \Lambda-\beta f(S) g(I)-\left(d_{S}+p\right) S+\varepsilon R\right) d t \\
& -f(S) g(I) \text { error }_{0} d t-S \text { error }_{1} d t
\end{aligned}
$$

$$
\begin{aligned}
d I= & \left(\beta f(S) g(I)-\left(d_{I}+\gamma\right) I\right) d t \\
& +f(S) g(I) \text { error }_{0} d t-I \text { error }_{2} d t, \\
d R= & \left(q \Lambda+p S+\gamma I-\left(d_{R}+\varepsilon\right) R\right) d t-R \text { error }_{3} d t .
\end{aligned}
$$

By the central limit theorem, the error term error ${ }_{i} d t(0 \leq$ $i \leq 3$ ) may be approximated by a normal distribution with zero mean and variance $\sigma_{i}^{2} d t(0 \leq i \leq 3)$, respectively. That is, error ${ }_{i} d t=\widetilde{N}\left(0, \sigma_{i}^{2} d t\right)$. Since these error ${ }_{i} d t$ may correlate with each other, we represent them by $l$-dimensional Brownian motion $B(t)=\left(B_{1}(t), \ldots, B_{l}(t)\right)$ as follows:

$$
\operatorname{error}_{i} d t=\sum_{j=1}^{l} \sigma_{i j} d B_{j}(t), \quad 0 \leq i \leq 3,
$$

where $\sigma_{i j}$ are all nonnegative real numbers. Therefore, model (4) is characterized by the following Itô stochastic differential equation:

$$
\begin{aligned}
d S= & \left((1-q) \Lambda-\beta f(S) g(I)-\left(d_{S}+P\right) S+\varepsilon R\right) d t \\
& -f(S) g(I) \sum_{j=1}^{l} \sigma_{0 j} d B_{j}(t)-S \sum_{j=1}^{l} \sigma_{1 j} d B_{j}(t), \\
d I= & \left(\beta f(S) g(I)-\left(d_{I}+\gamma\right) I\right) d t \\
& +f(S) g(I) \sum_{j=1}^{l} \sigma_{0 j} d B_{j}(t)-I \sum_{j=1}^{l} \sigma_{2 j} d B_{j}(t), \\
d R= & \left(q \Lambda+p S+\gamma I-\left(d_{R}+\varepsilon\right) R\right) d t \\
& -R \sum_{j=1}^{l} \sigma_{3 j} d B_{j}(t) .
\end{aligned}
$$

Model (6) in the special case where $f(S)=S, g(I)=I$, and $p=q=0$ has been investigated by Yang and Mao in [11] and in the special case where $\sigma_{1 j}=\sigma_{2 j}=\sigma_{3 j}=0(1 \leq j \leq l)$ and $p=q=0$ also has been discussed in [25]. It is well known that, in a stochastic epidemic model, the dynamical behaviors, like the extinction, persistence, stationary distribution, and stability of the model, are the most interesting topics. Therefore, in this paper, as an important extension and improvement of the results given in $[11,25]$, we aim to discuss the dynamical behaviors of model (6). Particularly, we will explore the stochastic extinction and persistence in the mean of disease with probability one and the existence of stationary distribution.

This paper is organized as follows. In Section 2, we introduce some preliminaries to be used in later sections. In Section 3, we establish the threshold condition for stochastic extinction of disease with probability one of model (6). In Section 4, we deduce the threshold conditions for the disease being stochastically persistent and permanent in the mean with probability one. In Section 5, we discuss the existence of the stationary distribution of model (6) under some sufficient conditions. In Section 6, the numerical simulations 
are presented to illustrate the main results obtained in this paper and some conjectures are further proposed. Finally, in Section 7 , a brief conclusion is given.

\section{Preliminaries}

Through this paper, we let $\left(\Omega, \mathscr{F},\left\{\mathscr{F}_{t}\right\}_{t \geq 0}, P\right)$ be a complete probability space with a filtration $\left\{\mathscr{F}_{t}\right\}_{t \geq 0}$ satisfying the usual conditions (that is, it is right continuous and increasing while $\mathscr{F}_{0}$ contains all $P$-null sets). In this paper, we always assume that stochastic model (6) is defined on probability space $\left(\Omega, \mathscr{F},\left\{\mathscr{F}_{t}\right\}_{t \geq 0}, P\right)$. Furthermore, we denote $R_{+}^{3}=\{(x, y, z)$ : $x>0, y>0, z>0\}, \sigma_{i}^{2}=\sum_{j=1}^{l} \sigma_{i j}^{2}, 0 \leq i \leq 3$, and $\sigma^{2}=\sum_{i=0}^{3} \sigma_{i}^{2}$.

Firstly, on the existence and uniqueness of global positive solution for model (4) we have the following result.

Lemma 1. Assume that $\left(\mathbf{H}_{\mathbf{1}}\right)$ and $\left(\mathbf{H}_{\mathbf{2}}\right)$ hold; then, for any initial value $(S(0), I(0), R(0)) \in R_{+}^{3}$, model (6) has a unique solution $(S(t), I(t), R(t))$ defined for all $t \geq 0$, and the solution will remain in $R_{+}^{3}$ with probability one.

This lemma can be proved by using a similar argument as in the proof of Theorem 3.1 given in [11]. We hence omit it here.

Lemma 2. Assume that $\left(\mathbf{H}_{\mathbf{1}}\right)$ and $\left(\mathbf{H}_{\mathbf{2}}\right)$ hold and let $(S(t), I(t), R(t))$ be the solution of model (6) with initial value $(S(0), I(0), R(0)) \in R_{+}^{3}$. Then $\lim \sup _{t \rightarrow \infty}(S(t)+I(t)+R(t))<$ $\infty$ a.s. Moreover, let $h(x, y, z)$ be any continuous function defined on $R_{+}^{3}$; then for each $1 \leq j \leq l$ we have

$$
\lim _{t \rightarrow \infty} \frac{1}{t} \int_{0}^{t} h(S(s), I(s), R(s)) d B_{j}(s)=0 .
$$

Proof. Let $N(t)=S(t)+I(t)+R(t)$; then we have from model (6)

$$
\begin{aligned}
d N(t) & =\left(\Lambda-d_{S} S(t)-d_{I} I(t)-d_{R} R(t)\right) d t-P(t) \\
& \leq(\Lambda-\mu N(t)) d t-P(t),
\end{aligned}
$$

where $\mu=\min \left\{d_{S}, d_{I}, d_{R}\right\}$ and $P(t)=\sum_{j=1}^{l}\left(\sigma_{1 j} S(t)+\sigma_{2 j} I(t)+\right.$ $\left.\sigma_{3 j} R(t)\right) d B_{j}(t)$. By the comparison theorem of stochastic differential equations, we further have

$$
N(t) \leq N(0) e^{-\mu t}+\frac{\Lambda}{\mu}\left(1-e^{-\mu t}\right)-Q(t),
$$

where

$$
\begin{aligned}
& Q(t)=\sum_{j=1}^{l} \int_{0}^{t} e^{-\mu(t-s)}\left(\sigma_{1 j} S(s)+\sigma_{2 j} I(s)\right. \\
& \left.+\sigma_{3 j} R(s)\right) d B_{j}(s) .
\end{aligned}
$$

Define $X(t)=N(0)+A(t)-U(t)-Q(t)$, where $A(t)=$ $(\Lambda / \mu)\left(1-e^{-\mu t}\right)$ and $U(t)=N(0)\left(1-e^{-\mu t}\right)$. It is clear that from Lemma 1 and (9) $X(t)$ is nonnegative for $t \geq 0$, and $A(t)$ and
$U(t)$ are continuous adapted increasing processes for $t \geq 0$ and $A(0)=U(0)=0$. Therefore, by Theorem 3.9 given in [27], we obtain that $\lim _{t \rightarrow \infty} X(t)<\infty$ a.s. exists. From (9), we further have

$$
\limsup _{t \rightarrow \infty} N(t)<\infty \text { a.s. }
$$

Denote

$$
M_{j}(t)=\int_{0}^{t} h(S(s), I(s), R(s)) d B_{j}(s) .
$$

By (11), we have

$$
\begin{aligned}
\frac{1}{t}\left\langle M_{j}, M_{j}\right\rangle(t) & =\frac{1}{t} \int_{0}^{t}(h(S(s), I(s), R(s)))^{2} d s \\
& \leq \sup _{t \geq 0}\left\{(h(S(t), I(t), R(t)))^{2}\right\}<\infty .
\end{aligned}
$$

Hence, the strong law of large number (see [27, 28]) implies $\lim _{t \rightarrow \infty}(1 / t) M_{j}(t)=0$ a.s. This completes the proof.

For any function $h(t)$ defined on $R_{+0}=[0,+\infty)$, we denote the average value on $[0, t]$ by $\langle h(t)\rangle=(1 / t) \int_{0}^{t} h(s) d s$.

Lemma 3. Assume that $\left(\mathbf{H}_{\mathbf{1}}\right)$ and $\left(\mathbf{H}_{\mathbf{2}}\right)$ hold. Let $(S(t), I(t)$, $R(t))$ be any positive solution of model (6); then

$$
\begin{aligned}
\langle S(t)\rangle= & \frac{\left[(1-q) d_{R}+\varepsilon\right] \Lambda}{d_{S}\left(d_{R}+\varepsilon\right)+p d_{R}} \\
& -\frac{d_{I}\left(d_{R}+\varepsilon\right)+d_{R} \gamma}{d_{S}\left(d_{R}+\varepsilon\right)+p d_{R}}\langle I(t)\rangle+\varphi(t),
\end{aligned}
$$

where function $\varphi(t)$ is defined for all $t \geq 0$ satisfying $\lim _{t \rightarrow \infty} \varphi(t)=0$.

Proof. Taking integration from 0 to $t$ for model (6), we get

$$
\begin{aligned}
& \frac{S(t)-S(0)}{t} \\
& =(1-q) \Lambda-\beta\langle S(t) g(I(t))\rangle-\left(d_{S}+p\right)\langle S(t)\rangle \\
& \quad+\varepsilon\langle R(t)\rangle \\
& \quad-\frac{1}{t} \int_{0}^{t} \sum_{j=1}^{l}\left(S(s) g(I(s)) \sigma_{0 j}+S(s) \sigma_{1 j}\right) d B_{j}(s), \\
& \frac{I(t)-I(0)}{t} \\
& \quad \beta\langle S(t) g(I(t))\rangle-\left(d_{I}+\gamma\right)\langle I(t)\rangle \\
& \quad+\frac{1}{t} \int_{0}^{t} \sum_{j=1}^{l}\left(S(s) g(I(s)) \sigma_{0 j}-I(s) \sigma_{2 j}\right) d B_{j}(s),
\end{aligned}
$$




$$
\begin{aligned}
& \frac{R(t)-R(0)}{t} \\
& =q \Lambda+\gamma\langle I(t)\rangle+p\langle S(t)\rangle-\left(d_{R}+\varepsilon\right)\langle R(t)\rangle \\
& \quad-\frac{1}{t} \int_{0}^{t} \sum_{j=1}^{l} R(s) \sigma_{3 j} d B_{j}(s) .
\end{aligned}
$$

Hence, we have

$$
\begin{aligned}
& \left(d_{R}+\varepsilon\right)\left(\frac{S(t)-S(0)}{t}+\frac{I(t)-I(0)}{t}\right)+\varepsilon \\
& . \frac{R(t)-R(0)}{t}=\left[(1-q) d_{R}+\varepsilon\right] \Lambda-\left(d_{S}\left(d_{R}+\varepsilon\right)\right. \\
& \left.+p d_{R}\right)\langle S(t)\rangle-\left[d_{I}\left(d_{R}+\varepsilon\right)+d_{R} \gamma\right]\langle I(t)\rangle-\frac{1}{t} \\
& \cdot \int_{0}^{t} \sum_{j=1}^{N}\left[\left(d_{R}+\varepsilon\right)\left(S(s) \sigma_{1 j}+I(s) \sigma_{2 j}\right)\right. \\
& \left.+\varepsilon R(s) \sigma_{3 j}\right] d B_{j}(s) .
\end{aligned}
$$

With a simple calculation from (16) we can easily obtain formula (14) with which $\varphi(t)$ is defined by

$$
\begin{aligned}
& \varphi(t)=-\frac{1}{d_{S}\left(d_{R}+\varepsilon\right)+p d_{R}}\left[\left(d_{R}+\varepsilon\right)\right. \\
& \cdot\left(\frac{S(t)-S(0)}{t}+\frac{I(t)-I(0)}{t}\right)+\varepsilon \frac{R(t)-R(0)}{t}+\frac{1}{t} \\
&\left.\quad \cdot \int_{0}^{t} \sum_{j=1}^{l}\left[\left(d_{R}+\varepsilon\right)\left(S(s) \sigma_{1 j}+I(s) \sigma_{2 j}\right)+\varepsilon R(s) \sigma_{3 j}\right] d B_{j}(s)\right] .
\end{aligned}
$$

By Lemma 2, we further have $\lim _{t \rightarrow \infty} \varphi(t)=0$ a.s.

Lemma 4. Assume that $\left(\mathbf{H}_{1}\right)$ and $\left(\mathbf{H}_{2}\right)$ hold and $\sigma_{1 j}=\sigma_{2 j}=$ $\sigma_{3 j}=0(1 \leq j \leq l)$. Then, for any solution $(S(t), I(t), R(t))$ of system (6) with $(S(0), I(0), R(0)) \in R_{+}^{3}$, one has

$$
\limsup _{t \rightarrow \infty}(S(t)+I(t)+R(t)) \leq \bar{S}, \text { a.s. }
$$

where $\bar{S}=\Lambda / \mu$. Furthermore, the region

$$
\Gamma=\{(S, I, R): S>0, I>0, R>0, S+I+R \leq \bar{S} \text { a.s. }\}
$$

is positive invariant with probability one for model (6), where $\mu=\min \left\{d_{S}, d_{I}, d_{R}\right\}$. have

In fact, for $N(t)=S(t)+I(t)+R(t)$, from model (6) we

$$
\begin{aligned}
d N(t) & =\left(\Lambda-d_{S} S(t)-d_{I} I(t)-d_{R} R(t)\right) d t \\
& \leq(\Lambda-\mu N(t)) d t, \text { a.s. }
\end{aligned}
$$

This implies that (18) holds, and set $\Gamma$ is positive invariant with probability one for model (6).
Lemma 5. Assume that $\left(\mathbf{H}_{1}\right)$ and $\left(\mathbf{H}_{2}\right)$ hold, $\sigma_{1 j}=\sigma_{2 j}=\sigma_{3 j}=$ $0(1 \leq j \leq l), d_{S}=d_{R}$, and $d_{I}=d_{S}+\alpha$ with constant $\alpha \geq 0$. Then, for any solution $(S(t), I(t), R(t))$ of model (6) with $(S(0), I(0), R(0)) \in R_{+}^{3}$, one has

$$
S(t)=\frac{\Lambda\left[(1-q) d_{S}+\varepsilon\right]}{d_{S}\left(d_{S}+\varepsilon+p\right)}+H(t)+G(t), \text { a.s. }
$$

$\forall t \geq 0$,

where

$$
\begin{aligned}
& H(t)=\frac{p \Lambda\left[(1-q) d_{S}+\varepsilon\right]}{d_{S}\left(d_{S}+\varepsilon\right)\left(d_{S}+\varepsilon+p\right)} e^{-\left(d_{S}+\varepsilon+p\right) t} \\
& -\left(R(0)-\frac{q \Lambda}{d_{S}+\varepsilon}\right) \frac{1}{p}\left(1-e^{-p t}\right) e^{-\left(d_{S}+\varepsilon\right) t} \\
& +\left(\frac{\Lambda}{d_{S}}-(S(0)+I(0)+R(0))\right) \\
& \cdot \frac{1}{p+\varepsilon}\left(1-e^{-(p+\varepsilon) t}\right) e^{-d_{S} t}, \\
& G(t)=-I(t)-\alpha \int_{0}^{t} e^{-d_{S}(t-s)} I(s) d s \\
& \quad-\gamma \int_{0}^{t} e^{-\left(d_{S}+\varepsilon\right)(t-s)} I(s) d s \\
& +p \int_{0}^{t} e^{-\left(d_{S}+\varepsilon+p\right)(t-s)} I(s) d s+p \alpha \int_{0}^{t} e^{-\left(d_{S}+\varepsilon+p\right)(t-s)} \\
& \cdot \int_{0}^{s} e^{-d_{S}(s-u)} I(u) d u d s+p \gamma \int_{0}^{t} e^{-\left(d_{S}+\varepsilon+p\right)(t-s)} \\
& \cdot \int_{0}^{s} e^{-\left(d_{S}+\varepsilon\right)(s-u)} I(u) d u d s .
\end{aligned}
$$

Proof. Since

$$
d N(t)=\left(\Lambda-d_{S} N(t)-\alpha I(t)\right) d t, \text { a.s., }
$$

then

$$
\begin{aligned}
N(t)= & \frac{\Lambda}{d_{S}}+\left(N(0)-\frac{\Lambda}{d_{S}}\right) e^{-d_{S} t} \\
& -\alpha \int_{0}^{t} e^{-d_{S}(t-s)} I(s) d s, \text { a.s., }
\end{aligned}
$$

where $N(0)=S(0)+I(0)+R(0)$. From the third equation of model (6) we have

$$
\begin{aligned}
R(t)= & \frac{q \Lambda}{d_{S}+\varepsilon}+\left(R(0)-\frac{q \Lambda}{d_{S}+\varepsilon}\right) e^{-\left(d_{S}+\varepsilon\right) t} \\
& +p \int_{0}^{t} e^{-\left(d_{S}+\varepsilon\right)(t-s)} S(s) d s \\
& +\gamma \int_{0}^{t} e^{-\left(d_{S}+\varepsilon\right)(t-s)} I(s) d s, \text { a.s. }
\end{aligned}
$$


Therefore,

$$
\begin{aligned}
S(t)= & \frac{\Lambda\left[(1-q) d_{S}+\varepsilon\right]}{d_{S}\left(d_{S}+\varepsilon\right)}-I(t) \\
& -\left(R(0)-\frac{q \Lambda}{d_{S}+\varepsilon}\right) e^{-\left(d_{S}+\varepsilon\right) t} \\
& +\left(N(0)-\frac{\Lambda}{d_{S}}\right) e^{-d_{S} t} \\
& -p \int_{0}^{t} e^{-\left(d_{S}+\varepsilon\right)(t-s)} S(s) d s \\
& -\alpha \int_{0}^{t} e^{-d_{S}(t-s)} I(s) d s \\
& -\gamma \int_{0}^{t} e^{-\left(d_{S}+\varepsilon\right)(t-s)} I(s) d s .
\end{aligned}
$$

Let $y(t)=\int_{0}^{t} e^{\left(d_{S}+\varepsilon\right) s} S(s) d s$; then

$$
\begin{aligned}
& d y(t)=\left[e^{\left(d_{S}+\varepsilon\right) t} S(t)\right] d t=[-p y(t) \\
& +\frac{\Lambda\left[(1-q) d_{S}+\varepsilon\right]}{d_{S}\left(d_{S}+\varepsilon\right)} e^{\left(d_{S}+\varepsilon\right) t}-I(t) e^{\left(d_{S}+\varepsilon\right) t} \\
& -\left(R(0)-\frac{q \Lambda}{d_{S}+\varepsilon}\right)+\left(N(0)-\frac{\Lambda}{d_{S}}\right) e^{(\varepsilon) t} \\
& \left.-\alpha e^{\varepsilon t} \int_{0}^{t} e^{d_{S} s} I(s) d s-\gamma \int_{0}^{t} e^{\left(d_{S}+\varepsilon\right) s} I(s) d s\right] d t
\end{aligned}
$$

Solving $y(t)$, we obtain

$$
\begin{aligned}
y(t) & =e^{-p t}\left[\frac{\Lambda\left[(1-q) d_{S}+\varepsilon\right]}{d_{S}\left(d_{S}+\varepsilon\right)\left(d_{S}+\varepsilon+p\right)}\left(e^{\left(d_{S}+\varepsilon+p\right) t}-1\right)\right. \\
& -\int_{0}^{t} e^{\left(d_{S}+\varepsilon+p\right) s} I(s) d s \\
& -\left(R(0)-\frac{q \Lambda}{d_{S}+\varepsilon}\right) \frac{1}{p}\left(e^{p t}-1\right) \\
& +\left(N(0)-\frac{\Lambda}{d_{S}}\right) \frac{1}{\varepsilon+p}\left(e^{(\varepsilon+p) t}-1\right) \\
& -\gamma \int_{0}^{t} e^{p s} \int_{0}^{s} e^{\left(d_{S}+\varepsilon\right) u} I(u) d u d s \\
& \left.-\alpha \int_{0}^{t} e^{\left(d_{S}+\varepsilon+p\right) s} \int_{0}^{s} e^{-d_{S}(s-u)} I(u) d s d s\right] d t
\end{aligned}
$$

Substituting (29) into (27), we immediately obtain (21)-(23). This completes the proof.

Remark 6. When $d_{S} \neq d_{R}$ in model (6), whether we can also establish a similar result as in Lemma 5 still is an interesting open problem.
Consider the following $n$-dimensional stochastic differential equation:

$$
d x(t)=b(x) d t+\sum_{r=1}^{m} \sigma_{r}(x) d B_{r}(t)
$$

where $x=\left(x_{1}, x_{2}, \ldots, x_{n}\right), \sigma_{r}(x)=\left(\sigma_{r}^{1}(x), \sigma_{r}^{2}(x), \ldots, \sigma_{r}^{n}(x)\right)$, and $B_{r}(t)(1 \leq r \leq m)$ are standard Brownian motions defined on the above probability space. The diffusion matrix is defined by

$$
\begin{gathered}
A(x)=\left(a_{i j}(x)\right)_{n \times n}, \\
a_{i j}(x)=\sum_{r=1}^{m} \sigma_{r}^{i}(x) \sigma_{r}^{j}(x) .
\end{gathered}
$$

For any second-order continuously differentiable function $V(x)$, we define

$$
L V(x)=\sum_{i=1}^{n} \frac{\partial V(x)}{\partial x_{i}} b_{i}(x)+\frac{1}{2} \sum_{i, j=1}^{n} \frac{\partial^{2} V(x)}{\partial x_{i} \partial x_{j}} a_{i j}(x) .
$$

The following lemma gives a criterion for the existence of stationary distribution in terms of Lyapunov function.

Lemma 7 (see [27]). Assume that there is a bounded open subset $D$ in $R^{n}$ with a regular (i.e., smooth) boundary such that

(i) there exist some $i=1,2, \ldots, n$ and positive constant $\eta>0$ such that $a_{i i}(x) \geq \eta$ for all $x \in D$;

(ii) there exists a nonnegative function $V(x): D^{c} \rightarrow R$ such that $V(x)$ is second-order continuously differentiable function and that, for some $\theta>0, L V(x) \leq-\theta$ for all $x \in D^{c}$, where $D^{c}=R^{n} \backslash D$.

Then (30) has a unique stationary distribution $\pi$. That is, if function $f$ is integrable with respect to the measure $\pi$, then for all $x_{0} \in R^{n}$

$$
\begin{aligned}
& P\left\{\lim _{t \rightarrow \infty} \frac{1}{t} \int_{0}^{t} f\left(x\left(u, x_{0}\right)\right) d u=\int_{R^{n}} f\left(x_{0}\right) \pi\left(d x_{0}\right)\right\} \\
& \quad=1 .
\end{aligned}
$$

To study the permanence in mean with probability one of model (6) we need the following result on the stochastic integrable inequality.

Lemma 8 (see [13]). Assume that functions $Y \in C\left(R_{+} \times \Omega, R_{+}\right)$ and $Z \in C\left(R_{+} \times \Omega, R_{+}\right)$satisfy $\lim _{t \rightarrow \infty}(Z(t) / t)=0$ a.s. If there is $T>0$ such that

$$
\ln Y(t) \geq v_{0} t-v \int_{0}^{t} Y(s) d s+Z(t) \text { a.s., }
$$

for all $t \geq T$, then

$$
\liminf _{t \rightarrow \infty} \frac{1}{t} \int_{0}^{t} Y(s) d s \geq \frac{v_{0}}{v} \text { a.s. }
$$




\section{Extinction of Disease}

For the convenience of following statements, we denote

$$
\begin{aligned}
& S_{0}=\frac{\left[(1-q) d_{R}+\varepsilon\right] \Lambda}{d_{S}\left(d_{R}+\varepsilon\right)+p d_{R}}, \\
& S_{1}=\frac{d_{I}\left(d_{R}+\varepsilon\right)+d_{R} \gamma}{d_{S}\left(d_{R}+\varepsilon\right)+p d_{R}} .
\end{aligned}
$$

We further define a threshold value

$$
\begin{aligned}
\widetilde{R}_{0}= & \frac{f\left(S_{0}\right) g^{\prime}(0)\left(\beta+\sum_{j=1}^{l} \sigma_{0 j} \sigma_{2 j}\right)}{d_{I}+\gamma} \\
& -\frac{\left(f\left(S_{0}\right) g^{\prime}(0)\right)^{2} \sigma_{0}^{2}}{2\left(d_{I}+\gamma\right)}-\frac{\sigma_{2}^{2}}{2\left(d_{I}+\gamma\right)} .
\end{aligned}
$$

Theorem 9. Assume that $\left(\mathbf{H}_{1}\right)$ and $\left(\mathbf{H}_{2}\right)$ hold. If one of the following conditions holds:

(a) $\widetilde{R}_{0}<1$ and $\sigma_{0}^{2} f\left(S_{0}\right) g^{\prime}(0) \leq \beta+\sum_{j=1}^{l} \sigma_{0 j} \sigma_{2 j}$,

(b) $\sigma_{0}>0$ and $\left(\beta+\sum_{j=1}^{l} \sigma_{0 j} \sigma_{2 j}\right)^{2} / 2 \sigma_{0}^{2}-\left(d_{I}+\gamma+(1 / 2) \sigma_{2}^{2}\right)<$ 0 ,

then, for any initial value $(S(0), I(0), R(0)) \in R_{+}^{3}$, one has

$$
\limsup _{t \rightarrow \infty} \frac{\ln I(t)}{t}<0 \text { a.s. }
$$

That is, disease I is stochastically extinct exponentially with probability one. Moreover,

$$
\begin{aligned}
& \lim _{t \rightarrow \infty}\langle S(t)\rangle=S_{0}, \\
& \lim _{t \rightarrow \infty}\langle R(t)\rangle=\frac{\Lambda\left(q d_{S}+p\right)}{d_{S}\left(d_{R}+\varepsilon\right)+p d_{R}} \text { a.s. }
\end{aligned}
$$

Proof. Applying Itô's formula to model (6) leads to

$$
\begin{aligned}
& \ln I(t)=\ln I(0)+\int_{0}^{t} f(x(s)) d s \\
& +\sum_{j=1}^{l}\left(\sigma_{0 j} \int_{0}^{t} f(S(s)) \frac{g(I(s))}{I(s)} d B_{j}(s)-\sigma_{2 j} B_{j}(t)\right),
\end{aligned}
$$

$$
-\left(d_{I}+\gamma+\frac{\sigma_{2}^{2}}{2}\right)-\frac{1}{2}\left(f(S) \frac{g(I)}{I}\right)^{2} \sigma_{0}^{2} .
$$

Assume that condition (b) holds. Since

$$
f(x(t)) \leq \frac{\left(\beta+\sum_{j=1}^{l} \sigma_{0 j} \sigma_{2 j}\right)^{2}}{2 \sigma_{0}^{2}}-\left(d_{I}+\gamma+\frac{1}{2} \sigma_{2}^{2}\right),
$$

then from $(40)$

$$
\begin{aligned}
& \frac{\ln I(t)}{t} \leq \frac{\ln I(0)}{t}+\frac{\left(\beta+\sum_{j=1}^{l} \sigma_{0 j} \sigma_{2 j}\right)^{2}}{2 \sigma_{0}^{2}}-\left(d_{I}+\gamma\right. \\
& \left.\quad+\frac{1}{2} \sigma_{2}^{2}\right)+\sum_{j=1}^{l}\left(\sigma_{0 j} \frac{1}{t} \int_{0}^{t} f(S(s)) \frac{g(I(s))}{I(s)} d B_{j}(s)\right. \\
& \left.-\sigma_{2 j} \frac{1}{t} \int_{0}^{t} d B_{j}(s)\right) .
\end{aligned}
$$

By Lemma 2, we have

$$
\begin{aligned}
\lim _{t \rightarrow \infty} \frac{1}{t} \int_{0}^{t} S(s) \frac{g(I(s))}{I(s)} d B_{j}(s) & =0 \\
\lim _{t \rightarrow \infty} \frac{1}{t} \int_{0}^{t} d B_{j}(s) & =0 \text { a.s., }
\end{aligned}
$$

$1 \leq j \leq l$.

Therefore,

$$
\begin{aligned}
\limsup _{t \rightarrow \infty} \frac{\ln I(t)}{t} \leq & \frac{\left(\beta+\sum_{j=1}^{l} \sigma_{0 j} \sigma_{2 j}\right)^{2}}{2 \sigma_{0}^{2}} \\
& -\left(d_{I}+\gamma+\frac{1}{2} \sigma_{2}^{2}\right)<0 .
\end{aligned}
$$

Assume that condition (a) holds. Choose constant $\epsilon>0$ such that $\beta+\sum_{j=1}^{l} \sigma_{0 j} \sigma_{2 j} \geq g^{\prime}(0) f(\epsilon) \sigma_{0}^{2}$. We compute that

$$
\begin{aligned}
= & f(S) \frac{g(I)}{I}\left(\beta+\sum_{j=1}^{l} \sigma_{0 j} \sigma_{2 j}\right)-\left(d_{I}+\gamma+\frac{\sigma_{2}^{2}}{2}\right) \\
& -\frac{1}{2}\left(f(S) \frac{g(I)}{I}\right)^{2} \sigma_{0}^{2} \\
= & f(S) \frac{g(I)}{I}\left(\beta+\sum_{j=1}^{l} \sigma_{0 j} \sigma_{2 j}\right)-\left(d_{I}+\gamma+\frac{\sigma_{2}^{2}}{2}\right) \\
& +\frac{1}{2} f^{2}(\epsilon) \frac{g^{2}(I)}{I^{2}} \sigma_{0}^{2} \\
& -\frac{1}{2}(f(S)-f(\epsilon))^{2} \frac{g^{2}(I)}{I^{2}} \sigma_{0}^{2} \\
& -f(S) f(\epsilon) \frac{g^{2}(I)}{I^{2}} \sigma_{0}^{2} \\
& -\left(d_{I}+\gamma+\frac{\sigma_{2}^{2}}{2}\right)+\frac{1}{2}\left(f(\epsilon) g^{\prime}(0)\right)^{2} \sigma_{0}^{2} . \\
\leq & \left.\left(\beta+\sum_{j=1}^{l} \sigma_{0 j} \sigma_{2 j}\right) \frac{g(I)}{I}-f(\epsilon) \sigma_{0}^{2} \frac{g^{2}(I)}{I^{2}}\right] f(S) \\
& {[(f)}
\end{aligned}
$$


When $\sigma_{0}^{2}=0$, which implies $\sigma_{0 j}=0(1 \leq j \leq l)$, we have from (46)

$$
\begin{aligned}
f(x) & \leq \beta f(S) \frac{g(I)}{I}-\left(d_{I}+\gamma+\frac{\sigma_{2}^{2}}{2}\right) \\
& \leq \beta g^{\prime}(0) f(S)-\left(d_{I}+\gamma+\frac{\sigma_{2}^{2}}{2}\right) .
\end{aligned}
$$

Since

$$
\begin{aligned}
f(S) & =f\left(S_{0}\right)+f(S)-f\left(S_{0}\right) \\
& =f\left(S_{0}\right)+f^{\prime}(\xi)\left(S-S_{0}\right),
\end{aligned}
$$

where $\xi \in\left(S, S_{0}\right)$, from $\left(H_{2}\right)$, we can obtain $f^{\prime}(\xi)\left(S-S_{0}\right) \leq$ $f^{\prime}\left(S_{0}\right)\left(S-S_{0}\right)$. Hence, we have

$$
f(S) \leq f\left(S_{0}\right)+f^{\prime}\left(S_{0}\right)\left(S-S_{0}\right) .
$$

According to (14), (40), and (49), we have

$$
\begin{aligned}
& \frac{\ln I(t)}{t} \leq \frac{\ln I(0)}{t}+\beta g^{\prime}(0) \frac{1}{t} \int_{0}^{t} f(S(s)) d s-\left(d_{I}\right. \\
& \left.+\gamma+\frac{\sigma_{2}^{2}}{2}\right)+\sum_{j=1}^{l}\left(\sigma_{0 j} \frac{1}{t} \int_{0}^{t} f(S(s)) \frac{g(I(s))}{I(s)} d B_{j}(s)\right. \\
& \left.-\sigma_{2 j} \frac{B_{j}(t)}{t}\right)=\frac{\ln I(0)}{t}+\beta g^{\prime}(0) f\left(S_{0}\right)-\beta g^{\prime}(0) \\
& \cdot f^{\prime}\left(S_{0}\right) S_{1}\langle I(t)\rangle+\beta f^{\prime}\left(S_{0}\right) g^{\prime}(0) \varphi(t)-\left(d_{I}+\gamma\right. \\
& \left.+\frac{\sigma_{2}^{2}}{2}\right)+\sum_{j=1}^{l}\left(\sigma_{0 j} \frac{1}{t} \int_{0}^{t} f(S(s)) \frac{g(I(s))}{I(s)} d B_{j}(s)\right. \\
& \left.-\sigma_{2 j} \frac{1}{t} \int_{0}^{t} d B_{j}(s)\right) .
\end{aligned}
$$

Hence, from (44) and Lemma 3, we finally have

$$
\begin{aligned}
& \limsup _{t \rightarrow \infty} \frac{\ln I(t)}{t} \leq \beta f\left(S_{0}\right) g^{\prime}(0)-\left(d_{I}+\gamma+\frac{\sigma_{2}^{2}}{2}\right) \text { a.s. } \\
& \text { When } \sigma_{0}^{2} \neq 0 \text {, from (40) and (46) we have } \\
& \frac{\ln I(t)}{t} \leq \frac{\ln I(0)}{t}+\frac{1}{t} \int_{0}^{t}\left[\left(\beta+\sum_{j=1}^{N} \sigma_{0 j} \sigma_{2 j}\right) \frac{g(I(s))}{I(s)}\right. \\
& \left.-f(\epsilon) \sigma_{0}^{2} \frac{g^{2}(I(s))}{I(s)^{2}}\right] f(S(s)) d s \\
& +\sum_{j=1}^{N}\left(\sigma_{0 j} \frac{1}{t} \int_{0}^{t} f(S(s)) \frac{g(I(s))}{I(s)} d B_{j}(s)\right. \\
& \left.-\sigma_{2 j} \frac{B_{j}(t)}{t}\right)-\left(d_{I}+\gamma+\frac{\sigma_{2}^{2}}{2}\right)+\frac{1}{2}\left(f(\epsilon) g^{\prime}(0)\right)^{2} \\
& \cdot \sigma_{0}^{2} .
\end{aligned}
$$

Define a function

$$
F(u)=\left(\beta+\sum_{j=1}^{l} \sigma_{0 j} \sigma_{2 j}\right) u-f(\epsilon) \sigma_{0}^{2} u^{2} .
$$

Clearly, $F(u)$ is a monotone increasing for $u \in[0,(\beta+$ $\left.\left.\sum_{j=1}^{l} \sigma_{0 j} \sigma_{2 j}\right) / 2 f(\epsilon) \sigma_{0}^{2}\right]$ and monotone decreasing for $u \in$ $\left[\left(\beta+\sum_{j=1}^{l} \sigma_{0 j} \sigma_{2 j}\right) / 2 f(\epsilon) \sigma_{0}^{2}, \infty\right)$. With condition $\beta+$ $\sum_{j=1}^{l} \sigma_{0 j} \sigma_{2 j} \geq g^{\prime}(0) f(\epsilon) \sigma_{0}^{2}$, that is, $g(I) / I \leq g^{\prime}(0) \leq(\beta+$ $\left.\sum_{j=1}^{l} \sigma_{0 j} \sigma_{2 j}\right) / 2 f(\epsilon) \sigma_{0}^{2}$, we have

$$
\begin{aligned}
F\left(\frac{g(I)}{I}\right) \leq & F\left(g^{\prime}(0)\right) \\
= & \left(\beta+\sum_{j=1}^{l} \sigma_{0 j} \sigma_{2 j}\right) g^{\prime}(0) \\
& -f(\epsilon) \sigma_{0}^{2}\left(g^{\prime}(0)\right)^{2} .
\end{aligned}
$$

Hence, by (14) and (49), we have

$$
\begin{aligned}
& \frac{\ln I(t)}{t} \leq \frac{\ln I(0)}{t}+g^{\prime}(0)\left(\beta+\sum_{j=1}^{l} \sigma_{0 j} \sigma_{2 j}-f(\epsilon)\right. \\
& \left.\cdot \sigma_{0}^{2} g^{\prime}(0)\right) \frac{1}{t} \int_{0}^{t} f(S(s)) d s \\
& +\sum_{j=1}^{l}\left(\sigma_{0 j} \frac{1}{t} \int_{0}^{t} f(S(s)) \frac{g(I(s))}{I(s)} d B_{j}(s)\right. \\
& \left.-\sigma_{2 j} \frac{1}{t} \int_{0}^{t} d B_{j}(s)\right)-\left(d_{I}+\gamma+\frac{\sigma_{2}^{2}}{2}\right)+\frac{1}{2}\left(g^{\prime}(0)\right. \\
& \cdot f(\epsilon))^{2} \sigma_{0}^{2} \leq \frac{\ln I(0)}{t}+g^{\prime}(0)\left(\beta+\sum_{j=1}^{l} \sigma_{0 j} \sigma_{2 j}\right. \\
& \left.-f(\epsilon) \sigma_{0}^{2} g^{\prime}(0)\right)\left[f\left(S_{0}\right)+f^{\prime}\left(S_{0}\right) \varphi(t)\right]-\left(d_{I}\right. \\
& \left.+\gamma+\frac{\sigma_{2}^{2}}{2}\right)+\frac{1}{2}\left(g^{\prime}(0) f(\epsilon)\right)^{2} \sigma_{0}^{2} \\
& +\sum_{j=1}^{l}\left(\sigma_{0 j} \frac{1}{t} \int_{0}^{t} S(s) \frac{g(I(s))}{I(s)} d B_{j}(s)\right. \\
& \left.+\int_{0}^{t} d B_{j}(s)\right) .
\end{aligned}
$$


Choose $\epsilon=S_{0}$; from (44) and Lemma 3, we finally have

$$
\begin{aligned}
\limsup _{t \rightarrow \infty} \frac{\ln I(t)}{t} \leq & f\left(S_{0}\right) g^{\prime}(0)\left(\beta+\sum_{j=1}^{l} \sigma_{0 j} \sigma_{2 j}\right) \\
& -\left(d_{I}+\gamma+\frac{\sigma_{2}^{2}}{2}\right) \\
& -\frac{1}{2}\left(f\left(S_{0}\right) g^{\prime}(0)\right)^{2} \sigma_{0}^{2} \text { a.s. }
\end{aligned}
$$

From (45), (51), and (56), it follows that (38) holds.

Since $\lim _{t \rightarrow \infty} I(t)=0$ a.s., by (14) of Lemma 3 and the last equation of (15), we further obtain

$$
\begin{aligned}
& \lim _{t \rightarrow \infty}\langle S(t)\rangle=S_{0}, \\
& \lim _{t \rightarrow \infty}\langle R(t)\rangle=\frac{\Lambda\left(q d_{S}+p\right)}{d_{S}\left(d_{R}+\varepsilon\right)+p d_{R}} \text { a.s. }
\end{aligned}
$$

This completes the proof.

Remark 10. Condition (b) in Theorem 9 can be rewritten in the following form:

$$
\sigma_{0}^{2}>\frac{\left(\beta+\sum_{j=1}^{l} \sigma_{0 j} \sigma_{2 j}\right)^{2}}{2\left(d_{I}+\gamma+(1 / 2) \sigma_{2}^{2}\right)} .
$$

It is clear that

$$
\begin{gathered}
\frac{f\left(S_{0}\right) g^{\prime}(0)\left(\beta+\sum_{j=1}^{l} \sigma_{0 j} \sigma_{2 j}\right)}{d_{I}+\gamma+(1 / 2) \sigma_{2}^{2}} \\
-\frac{\left(f\left(S_{0}\right) g^{\prime}(0)\right)^{2}\left(\beta+\sum_{j=1}^{l} \sigma_{0 j} \sigma_{2 j}\right)^{2}}{4\left(d_{I}+\gamma+(1 / 2) \sigma_{2}^{2}\right)^{2}} \leq 1 .
\end{gathered}
$$

Therefore, when condition (b) holds, from (58) we also have

$$
\begin{aligned}
\widetilde{R}_{0} & =\frac{f\left(S_{0}\right) g^{\prime}(0)\left(\beta+\sum_{j=1}^{l} \sigma_{0 j} \sigma_{2 j}\right)}{d_{I}+\gamma} \\
& -\frac{\left(f\left(S_{0}\right) g^{\prime}(0)\right)^{2} \sigma_{0}^{2}}{2\left(d_{I}+\gamma\right)}-\frac{\sigma_{2}^{2}}{2\left(d_{I}+\gamma\right)} \\
& <\frac{f\left(S_{0}\right) g^{\prime}(0)\left(\beta+\sum_{j=1}^{l} \sigma_{0 j} \sigma_{2 j}\right)}{d_{I}+\gamma} \\
- & \frac{\left(f\left(S_{0}\right) g^{\prime}(0)\right)^{2}\left(\beta+\sum_{j=1}^{l} \sigma_{0 j} \sigma_{2 j}\right)^{2}}{4\left(d_{I}+\gamma\right)\left(d_{I}+\gamma+(1 / 2) \sigma_{2}^{2}\right)} \\
- & \frac{\sigma_{2}^{2}}{2\left(d_{I}+\gamma\right)}=\left[\frac{f\left(S_{0}\right) g^{\prime}(0)\left(\beta+\sum_{j=1}^{l} \sigma_{0 j} \sigma_{2 j}\right)}{d_{I}+\gamma+(1 / 2) \sigma_{2}^{2}}\right.
\end{aligned}
$$

$$
\begin{aligned}
& \left.-\frac{\left(f\left(S_{0}\right) g^{\prime}(0)\right)^{2}\left(\beta+\sum_{j=1}^{l} \sigma_{0 j} \sigma_{2 j}\right)^{2}}{4\left(d_{I}+\gamma+(1 / 2) \sigma_{2}^{2}\right)^{2}}\right] \\
& \times \frac{d_{I}+\gamma+(1 / 2) \sigma_{2}^{2}}{d_{I}+\gamma}-\frac{\sigma_{2}^{2}}{2\left(d_{I}+\gamma\right)} \leq 1 .
\end{aligned}
$$

Remark 11. From Remark 10 above, we see that in Theorem 9 if condition (a) holds, then we directly have $\widetilde{R}_{0}<1$, and if condition (b) holds, then we also have $\widetilde{R}_{0}<1$. Therefore, an interesting open problem is whether we can establish the extinction of disease $I$ with probability one for model (6) only when $\widetilde{R}_{0}<1$.

\section{Stochastic Persistence in the Mean}

In this section, we discuss the stochastic persistence and permanence in the mean with probability one for model (6) only for the following two special cases: (1) $\sigma_{0 j}=0(1 \leq j \leq l)$ and (2) $\sigma_{1 j}=\sigma_{2 j}=\sigma_{3 j}=0(1 \leq j \leq l)$. Furthermore, we also assume that in model (6) function $f(S) \equiv S$.

4.1. Case $\sigma_{0 j}=0(1 \leq j \leq l)$. When $f(S)=S$ and $\sigma_{0 j}=$ $0(1 \leq j \leq l)$ in model $(6)$, we have

$$
\widetilde{R}_{0}=\frac{S_{0} g^{\prime}(0) \beta}{d_{I}+\gamma}-\frac{\sigma_{2}^{2}}{2\left(d_{I}+\gamma\right)} .
$$

Theorem 12. Assume that $\left(\mathbf{H}_{1}\right)$ holds, $f(S)=S$, and $\sigma_{0 j}=$ $0(1 \leq j \leq l)$. If $\widetilde{R}_{0}>1$; then disease I in model (6) is stochastically persistent in the mean; that is,

$$
\liminf _{t \rightarrow \infty} \frac{1}{t} \int_{0}^{t} I(r) d r>0 \text { a.s. }
$$

Proof. Let $(S(t), I(t), R(t))$ be any positive solution of model (6). Lemma 2 implies that there is a constant $M_{0}>0$ such that $S(t)+I(t)+R(t) \leq M_{0}$ a.s. for all $\geq 0$. Define a Lyapunov function

$$
U(I)=\int_{I(0)}^{I(t)} \frac{1}{g(I)} d I .
$$

Using Itô's formula to model (6) leads to

$$
\begin{aligned}
& d U(I)=\left(\beta S-\left(d_{I}+\gamma\right) \frac{I}{g(I)}-\frac{\sigma_{2}^{2}}{2} \frac{I^{2}}{g^{2}(I)} g^{\prime}(I)\right) d t \\
& -\sum_{j=1}^{l} \sigma_{2 j} \frac{I}{g(I)} d B_{j}(t)=(\beta S \\
& -\left(d_{I}+\gamma\right)\left(\frac{I}{g(I)}-\frac{1}{g^{\prime}(0)}\right) \\
& -\frac{\sigma_{2}^{2}}{2}\left(\frac{I^{2}}{g^{2}(I)} g^{\prime}(I)-\frac{1}{g^{\prime}(0)}\right) \\
& \left.-\left(\frac{d_{I}+\gamma}{g^{\prime}(0)}+\frac{\sigma_{2}^{2}}{2 g^{\prime}(0)}\right)\right) d t-\sum_{j=1}^{l} \sigma_{2 j} \frac{I}{g(I)} d B_{j}(t) .
\end{aligned}
$$


From $\left(H_{1}\right)$, which implies that $g^{\prime}(I) \leq g(I) / I \leq g^{\prime}(0)$, we have

$$
\begin{aligned}
& \frac{I^{2}}{g^{2}(I)} g^{\prime}(I)-\frac{1}{g^{\prime}(0)} \\
& =\frac{I^{2}}{g^{2}(I)}\left(g^{\prime}(I)-g^{\prime}(0)\right) \\
& \quad+g^{\prime}(0)\left(\frac{I^{2}}{g^{2}(I)}-\frac{1}{\left(g^{\prime}(0)\right)^{2}}\right) \\
& \leq g^{\prime}(0)\left(\frac{I}{g(I)}+\frac{1}{g^{\prime}(0)}\right)\left(\frac{I}{g(I)}-\frac{1}{g^{\prime}(0)}\right) \\
& \leq\left(M g^{\prime}(0)+1\right)\left(\frac{I}{g(I)}-\frac{1}{g^{\prime}(0)}\right),
\end{aligned}
$$

where $M=\sup _{0 \leq I \leq M_{0}}\{I / g(I)\}$. Since $\lim _{I \rightarrow 0^{+}}(I / g(I))=$ $1 / g^{\prime}(0)$, then $0<M<\infty$. Substituting (65) into (64) and then integrating from 0 to $t \geq 0$, we get

$$
\begin{aligned}
& \frac{U(I)}{t} \geq \frac{1}{t} \int_{0}^{t}\left[\beta S(r)-\left(d_{I}+\gamma+\frac{\sigma_{2}^{2}}{2}\left(M g^{\prime}(0)+1\right)\right)\right. \\
& \left.\cdot\left(\frac{I(r)}{g(I(r))}-\frac{1}{g^{\prime}(0)}\right)-\left(\frac{d_{I}+\gamma}{g^{\prime}(0)}+\frac{\sigma_{2}^{2}}{2 g^{\prime}(0)}\right)\right] d r \\
& \quad-\sum_{j=1}^{l} \frac{1}{t} M_{j}(t),
\end{aligned}
$$

where $M_{j}(t)=\int_{0}^{t} \sigma_{2 j}(I(r) / g(I(r))) d B_{j}(r)$. From Lemma 2, we have

$$
\lim _{t \rightarrow \infty} \frac{1}{t} M_{j}(t)=0 \text { a.s., } \quad 1 \leq j \leq l .
$$

Define a function $G(I)$ as follows. When $I>0, G(I)=$ $I / g(I)$, and when $I=0, G(0)=\lim _{I \rightarrow 0}(I / g(I))=1 / g^{\prime}(0)$. Then $G(I)$ is continuous for $I \geq 0$ and differentiable for $I>0$. Applying Lagrange's mean value theorem to $G(I)-G(0)$, we have from $(66)$

$$
\frac{U(I)}{t} \geq \frac{1}{t} \int_{0}^{t}\left[\beta S(r)-\left(d_{I}+\gamma+\frac{\sigma_{2}^{2}}{2}\left(M g^{\prime}(0)+1\right)\right)\right.
$$

$$
\begin{aligned}
& \left.\cdot \sup _{0 \leq I \leq M_{0}}\left\{G^{\prime}(I)\right\} I(r)-\left(\frac{d_{I}+\gamma}{g^{\prime}(0)}+\frac{\sigma_{2}^{2}}{2 g^{\prime}(0)}\right)\right] d r \\
& -\sum_{j=1}^{l} \frac{1}{t} M_{j}(t),
\end{aligned}
$$

Substituting (14) into (68), it follows that

$$
\begin{aligned}
& \frac{U(I)}{t} \geq \beta S_{0}-\left[\beta S_{1}\right. \\
& \left.+\left(d_{I}+\gamma+\frac{\sigma_{2}^{2}}{2}\left(M g^{\prime}(0)+1\right)\right) \sup _{0 \leq I \leq M_{0}}\left\{G^{\prime}(I)\right\}\right] \\
& \cdot\langle I(t)\rangle-\left(\frac{d_{I}+\gamma}{g^{\prime}(0)}+\frac{\sigma_{2}^{2}}{2 g^{\prime}(0)}\right)-\sum_{j=1}^{l} \frac{1}{t} M_{j}(t) \\
& +\beta \varphi(t) .
\end{aligned}
$$

Since

$$
\begin{aligned}
\frac{U(I)}{t} & =\frac{1}{t} \int_{I(0)}^{I(t)} \frac{I}{g(I)} \frac{1}{I} d I \leq \frac{1}{t} \int_{I(0)}^{I(t)} M \frac{1}{I} d I \\
& =M \frac{\ln I(t)-\ln I(0)}{t},
\end{aligned}
$$

we have

$$
\begin{aligned}
& \frac{\ln I(t)}{t} \geq \frac{1}{M}\left\{\beta S_{0}-\frac{d_{I}+\gamma}{g^{\prime}(0)}-\frac{\sigma_{2}^{2}}{2 g^{\prime}(0)}\right\}-\frac{1}{M}\left[\beta S_{1}\right. \\
& \left.+\left(d_{I}+\gamma+\frac{\sigma_{2}^{2}}{2}\left(M g^{\prime}(0)+1\right)\right) \sup _{0 \leq I \leq M_{0}}\left\{G^{\prime}(I)\right\}\right] \\
& \cdot\langle I(t)\rangle+\Phi(t),
\end{aligned}
$$

where

$$
\Phi(t)=-\frac{1}{M} \sum_{j=1}^{l} \frac{1}{t} M_{j}(t)+\frac{\beta \varphi(t)}{M}+\frac{1}{t} \ln I(0) .
$$

From (67) and Lemma 3 we have $\lim _{t \rightarrow \infty} \Phi(t)=0$. Finally, by Lemma 8 , we obtain

$$
\liminf _{t \rightarrow \infty} \frac{1}{t} \int_{0}^{t} I(r) d r \geq I^{*}
$$

where

$$
I^{*}=\frac{\left(d_{I}+\gamma\right)\left(\widetilde{R}_{0}-1\right)}{\left(\beta S_{1}+\left(d_{I}+\gamma+(1 / 2) \sigma_{2}^{2}\left(M g^{\prime}(0)+1\right)\right) \sup _{0 \leq I \leq M_{0}}\left\{G^{\prime}(I)\right\}\right) g^{\prime}(0)} .
$$


in (74) are dependent on every solution $(S(t), I(t), R(t))$ of model (6). This shows that in Theorem 12 we only obtain the stochastic persistence in the mean of the disease.

4.2. Case $\sigma_{1 j}=\sigma_{2 j}=\sigma_{3 j}=0(1 \leq j \leq l)$. When $f(S)=S$ and $\sigma_{1 j}=\sigma_{2 j}=\sigma_{3 j}=0(1 \leq j \leq l)$ in model (6), we have

$$
\widetilde{R}_{0}=\frac{\beta S_{0} g^{\prime}(0)}{d_{I}+\gamma}-\frac{S_{0}^{2}\left(g^{\prime}(0)\right)^{2} \sigma_{0}^{2}}{2\left(d_{I}+\gamma\right)} .
$$

In order to obtain the stochastic permanence in the mean with probability one for model (6), we need to introduce a new threshold value

$$
\bar{R}_{0}=\frac{\beta S_{0} g^{\prime}(0)}{d_{I}+\gamma}-\frac{\bar{s}^{2}\left(g^{\prime}(0)\right)^{2} \sigma_{0}^{2}}{2\left(d_{I}+\gamma\right)} .
$$

Obviously, we have $\bar{R}_{0} \leq \widetilde{R}_{0}$.

Theorem 14. Assume that $\left(\mathbf{H}_{1}\right)$ holds, $f(S)=S$, and $\sigma_{1 j}=$ $\sigma_{2 j}=\sigma_{3 j}=0(1 \leq j \leq l)$. If $\bar{R}_{0}>1$, then disease I in model (6) is stochastically permanent in the mean, that is,

$$
\begin{aligned}
& \liminf _{t \rightarrow \infty} \frac{1}{t} \int_{0}^{t} I(r) d r \\
& \geq \frac{\left(d_{I}+\gamma\right)\left(\bar{R}_{0}-1\right)}{\left(\beta S_{1}+\left(d_{I}+\gamma\right) \max _{0 \leq I \leq \bar{S}} G^{\prime}(I)\right) g^{\prime}(0)} \text { a.s., }
\end{aligned}
$$

where function $G(I)$ is defined in Theorem 12 above.

Proof. Let $U(I)=\int_{I(0)}^{I(t)}(1 / g(I)) d I$; using Itô's formula to model (6) and (18) leads to

$$
\begin{aligned}
& d U(I)=\left(\beta S-\left(d_{I}+\gamma\right) \frac{I}{g(I)}-\frac{\sigma_{0}^{2}}{2} S^{2} g^{\prime}(I)\right) d t \\
& -\sum_{j=1}^{l} S \sigma_{0 j} d B_{j}(t) \geq(\beta S \\
& -\left(d_{I}+\gamma\right)\left(\frac{I}{g(I)}-\frac{1}{g^{\prime}(0)}\right) \\
& \left.-\left(\frac{\sigma_{0}^{2} \bar{S}^{2} g^{\prime}(0)}{2}+\frac{d_{I}+\gamma}{g^{\prime}(0)}\right)\right) d t-\sum_{j=1}^{l} S \sigma_{0 j} d B_{j}(t) .
\end{aligned}
$$

Similarly to above proof of Theorem 12, we have

$$
\begin{aligned}
& \frac{U(I)}{t} \geq \frac{1}{t} \int_{0}^{t}\left(\beta S(r)-\left(d_{I}+\gamma\right) \max _{0 \leq I \leq \bar{S}} G^{\prime}(I) I(r)\right. \\
& \left.-\left(\frac{\sigma_{0}^{2} \bar{S}^{2} g^{\prime}(0)}{2}+\frac{d_{I}+\gamma}{g^{\prime}(0)}\right)\right) d r \\
& -\sum_{j=1}^{l} \int_{0}^{t} S(r) \sigma_{0 j} d B_{j}(r) .
\end{aligned}
$$

Substituting (14) into (79) yields

$$
\begin{aligned}
\frac{U(I)}{t} \geq & \beta S_{0} \\
& -\left[\beta S_{1}+\left(d_{I}+\gamma\right) \max _{0 \leq I \leq \bar{S}} G^{\prime}(I)\right] \frac{1}{t} \int_{0}^{t} I(r) d r \\
& -\left(\frac{\sigma_{0}^{2} \bar{S}^{2} g^{\prime}(0)}{2}+\frac{d_{I}+\gamma}{g^{\prime}(0)}\right) \\
& -\sum_{j=1}^{l} \frac{1}{t} \int_{0}^{t} S(r) \sigma_{0 j} d B_{j}(r)+\beta \varphi(t) .
\end{aligned}
$$

Since by (18)

$$
\frac{U(I)}{t} \leq \frac{1}{t} \int_{I(0)}^{I(t)} \frac{\bar{S}}{g(\bar{S})} \frac{1}{I} d I \leq \frac{\bar{S}(\ln \bar{S}-\ln I(0))}{g(\bar{S}) t},
$$

(80) can be rewritten as

$$
\begin{aligned}
& \frac{1}{t} \int_{0}^{t} I(r) d r \\
& \quad \geq \frac{\left(d_{I}+\gamma\right)\left(\bar{R}_{0}-1\right)+g^{\prime}(0) \Phi(t)}{\left(\beta S_{1}+\left(d_{I}+\gamma\right) \max _{0 \leq I \leq \bar{S}} G^{\prime}(I)\right) g^{\prime}(0)},
\end{aligned}
$$

where

$$
\begin{gathered}
\Phi(t)=-\sum_{j=1}^{l} \frac{1}{t} \int_{0}^{t} S(r) \sigma_{0 j} d B_{j}(r)+\beta \varphi(t) \\
-\frac{\bar{S}(\ln \bar{S}-\ln I(0))}{g(\bar{S}) t} .
\end{gathered}
$$

By Lemmas 2 and 3, it follows that $\lim _{t \rightarrow \infty} \Phi(t)=0$. Therefore, taking $t \rightarrow \infty$ in (82) it follows that (77) holds. This completes the proof.

Using Lemma 5, we can establish the following result which shows that $\widetilde{R}_{0}$ can be a threshold value for the stochastic permanence of disease $I$ in the mean for a more special case of model (6): $d_{S}=d_{R}$ and $d_{I}=d_{S}+\alpha$ with constant $\alpha \geq 0$.

Theorem 15. Assume that $\left(\mathbf{H}_{1}\right)$ holds, $f(S)=S, \sigma_{1 j}=\sigma_{2 j}=$ $\sigma_{3 j}=0(1 \leq j \leq l), d_{S}=d_{R}$, and $d_{I}=d_{S}+\alpha$ with constant $\alpha \geq 0$. If $\widetilde{R}_{0}>1$; then disease I in model (6) is stochastically permanent in the mean; that is,

$$
\begin{aligned}
& \liminf _{t \rightarrow \infty} \frac{1}{t} \int_{0}^{t} I(r) d r \\
& \geq \frac{\left(d_{S}+\alpha+\gamma\right)\left(\widetilde{R}_{0}-1\right)}{\left(\beta S_{1}+\left(d_{I}+\gamma\right) \max _{0 \leq I \leq \bar{S}} G^{\prime}(I)+M_{0}\right) g^{\prime}(0)} \text { a.s., }
\end{aligned}
$$


where function $G(I)$ is defined in above Theorem 12 and

$$
\begin{aligned}
M_{0} & =6\left[1+\frac{\alpha^{2}}{d_{S}^{2}}+\frac{\gamma^{2}}{\left(d_{S}+\varepsilon\right)^{2}}+\frac{p^{2}}{\left(d_{S}+\varepsilon+p\right)^{2}}\right. \\
& \left.+\frac{(p \alpha)^{2}}{d_{S}^{2}\left(d_{S}+\varepsilon+p\right)^{2}}+\frac{(p \gamma)^{2}}{\left(d_{S}+\varepsilon\right)^{2}\left(d_{S}+\varepsilon+p\right)^{2}}\right] \frac{\Lambda}{d_{S}} .
\end{aligned}
$$

Proof. Firstly, when $d_{S}=d_{R}$ and $d_{I}=d_{S}+\alpha$, then threshold value $\widetilde{R}_{0}$ becomes

$$
\widetilde{R}_{0}=\frac{\beta S_{0} g^{\prime}(0)}{d_{S}+\alpha+\gamma}-\frac{S_{0}^{2}\left(g^{\prime}(0)\right)^{2} \sigma_{0}^{2}}{2\left(d_{S}+\alpha+\gamma\right)}
$$

where $S_{0}=\Lambda\left[(1-q) d_{S}+\varepsilon\right] / d_{S}\left(d_{S}+\varepsilon+p\right)$.

Let $U(I)=\int_{I(0)}^{I(t)}(1 / g(I)) d I$; similarly to above proof of Theorem 12, we have

$$
\begin{aligned}
& d U(I) \geq\left(\beta S-\left(d_{S}+\alpha+\gamma\right) \max _{0 \leq I \leq \bar{S}} G^{\prime}(I) I\right. \\
& \left.-\frac{1}{2} \sigma_{0}^{2} S^{2} g^{\prime}(0)-\frac{d_{S}+\alpha+\gamma}{g^{\prime}(0)}\right) d t \\
& -\sum_{j=1}^{l} S \sigma_{0 j} d B_{j}(t) .
\end{aligned}
$$

Since $S^{2}=S_{0}^{2}+2 S_{0}\left(S-S_{0}\right)+\left(S-S_{0}\right)^{2}$, we further have

$$
\begin{aligned}
\frac{U(I)}{t} \geq & \beta\langle S(t)\rangle-\left(d_{S}+\alpha+\gamma\right) \max _{0 \leq I \leq \bar{S}} G^{\prime}(I)\langle I(t)\rangle \\
& -\frac{1}{2} \sigma_{0}^{2} S_{0}^{2} g^{\prime}(0)-\sigma_{0}^{2} S_{0} g^{\prime}(0)\left\langle S(t)-S_{0}\right\rangle \\
& -\frac{d_{S}+\alpha+\gamma}{g^{\prime}(0)}-\frac{1}{2} \sigma_{0}^{2} g^{\prime}(0)\left\langle\left(S(t)-S_{0}\right)^{2}\right\rangle \\
& -\sum_{j=1}^{l} \frac{1}{t} \int_{0}^{t} S \sigma_{0 j} d B_{j}(s) .
\end{aligned}
$$

Substituting (14) and (21) of Lemma 5 into (88), using inequality $(a+b)^{2} \leq 2\left(a^{2}+b^{2}\right)$, it follows that

$$
\begin{aligned}
\frac{U(I)}{t} \geq & \frac{d_{S}+\alpha+\gamma}{g^{\prime}(0)}\left(\widetilde{R}_{0}-1\right) \\
& -\left[\beta S_{1}+\left(d_{S}+\alpha+\gamma\right) \max _{0 \leq I \leq \bar{S}} G^{\prime}(I)\right]\langle I(t)\rangle \\
& +\left(\beta-\sigma_{0}^{2} S_{0} g^{\prime}(0)\right) \varphi(t) \\
& -\sigma_{0}^{2} g^{\prime}(0)\left(\left\langle H^{2}(t)\right\rangle+\left\langle G^{2}(t)\right\rangle\right) \\
& -\sum_{j=1}^{l} \frac{1}{t} \int_{0}^{t} S \sigma_{0 j} d B_{j}(s) .
\end{aligned}
$$

From expression (22) of $H(t)$, we easily have $\lim _{t \rightarrow \infty}\left\langle H^{2}(t)\right\rangle=0$. By (18), without loss of generality, we can assume that $S(t)+I(t)+R(t) \leq \Lambda / d_{S}$ a.s. for all $t \geq 0$. Hence,

$$
\begin{aligned}
& G^{2}(t) \leq 6\left[I^{2}(t)+\alpha^{2}\left(\int_{0}^{t} e^{-d_{s}(t-s)} I(s) d s\right)^{2}\right. \\
& +\gamma^{2}\left(\int_{0}^{t} e^{-\left(d_{S}+\varepsilon\right)(t-s)} I(s) d s\right)^{2} \\
& +p^{2}\left(\int_{0}^{t} e^{-\left(d_{S}+\varepsilon+p\right)(t-s)} I(s) d s\right)^{2}+(p \alpha)^{2} \\
& \cdot\left(\int_{0}^{t} e^{-\left(d_{S}+\varepsilon+p\right)(t-s)} \int_{0}^{s} e^{-d_{S}(s-u)} I(u) d u d s\right)^{2} \\
& +(p \gamma)^{2}\left(\int_{0}^{t} e^{-\left(d_{S}+\varepsilon+p\right)(t-s)}\right. \\
& \left.\left.\cdot \int_{0}^{s} e^{-\left(d_{S}+\varepsilon\right)}(s-u) I(u) d u d s\right)^{2}\right] \leq 6\left[\frac{\Lambda}{d_{S}} I(t)\right. \\
& +\frac{\alpha^{2} \Lambda}{d_{S}^{2}} \int_{0}^{t} e^{-d_{S}(t-s)} I(s) d s+\frac{\gamma^{2} \Lambda}{d_{S}\left(d_{S}+\varepsilon\right)} \\
& \cdot \int_{0}^{t} e^{-\left(d_{S}+\varepsilon\right)(t-s)} I(s) d s+\frac{p^{2} \Lambda}{d_{S}\left(d_{S}+\varepsilon+p\right)} \\
& \cdot \int_{0}^{t} e^{-\left(d_{S}+\varepsilon+p\right)(t-s)} I(s) d s+\frac{(p \alpha)^{2} \lambda}{d_{S}^{2}\left(d_{S}+\varepsilon+p\right)} \\
& \cdot \int_{0}^{t} e^{-\left(d_{S}+\varepsilon+p\right)(t-s)} \int_{0}^{s} e^{-d_{S}(s-u)} I(u) d u d s \\
& +\frac{(p \gamma)^{2} \Lambda}{d_{S}\left(d_{S}+\varepsilon\right)\left(d_{S}+\varepsilon+p\right)} \int_{0}^{t} e^{-\left(d_{S}+\varepsilon+p\right)(t-s)} \\
& \left.\cdot \int_{0}^{s} e^{-\left(d_{s}+\varepsilon\right)}(s-u) I(u) d u d s\right] \text {. }
\end{aligned}
$$

By computing, we obtain

$$
\begin{aligned}
& \frac{1}{t} \int_{0}^{t} \int_{0}^{s} e^{-d_{S}(s-u)} I(u) d u d s \leq \frac{1}{d_{S}}\langle I(t)\rangle \\
& \frac{1}{t} \int_{0}^{t} \int_{0}^{s} e^{-\left(d_{S}+\varepsilon\right)(s-u)} I(u) d u d s \leq \frac{1}{d_{S}+\varepsilon}\langle I(t)\rangle \\
& \frac{1}{t} \int_{0}^{t} \int_{0}^{s} e^{-\left(d_{S}+\varepsilon+p\right)(s-u)} I(u) d u d s \leq \frac{1}{d_{S}+\varepsilon+p}\langle I(t)\rangle, \\
& \frac{1}{t} \int_{0}^{t} \int_{0}^{s} e^{-\left(d_{S}+\varepsilon+p\right)(s-u)} \int_{0}^{u} e^{-d_{S}(u-v)} I(v) d v d u d s \\
& \quad \leq \frac{1}{d_{S}\left(d_{S}+\varepsilon+p\right)}\langle I(t)\rangle
\end{aligned}
$$




$$
\begin{aligned}
& \frac{1}{t} \int_{0}^{t} \int_{0}^{s} e^{-\left(d_{S}+\varepsilon+p\right)(s-u)} \int_{0}^{u} e^{-\left(d_{S}+\varepsilon\right)(u-v)} I(v) d v d u d s \\
& \quad \leq \frac{1}{\left(d_{S}+\varepsilon\right)\left(d_{S}+\varepsilon+p\right)}\langle I(t)\rangle
\end{aligned}
$$

Therefore, we finally have

$$
\left\langle G^{2}(t)\right\rangle \leq M_{0}\langle I(t)\rangle
$$

From (81), (89), and (92) we further obtain

$$
\begin{aligned}
& \frac{1}{t} \int_{0}^{t} I(r) d r \\
& \quad \geq \frac{\left(d_{S}+\alpha+\gamma\right)\left(\widetilde{R}_{0}-1\right)+g^{\prime}(0) \Phi(t)}{\left(\beta S_{1}+\left(d_{I}+\gamma\right) \max _{0 \leq I \leq \bar{S}} G^{\prime}(I)+M_{0}\right) g^{\prime}(0)},
\end{aligned}
$$

where

$$
\begin{aligned}
\Phi(t)= & -\sum_{j=1}^{l} \frac{1}{t} \int_{0}^{t} S(r) \sigma_{0 j} d B_{j}(r) \\
& +\left(\beta-\sigma_{0}^{2} S_{0} g^{\prime}(0)\right) \varphi(t)-\sigma_{0}^{2} g^{\prime}(0)\left\langle H^{2}(t)\right\rangle \\
& -\frac{\bar{S}(\ln \bar{S}-\ln I(0))}{g(\bar{S}) t} .
\end{aligned}
$$

By Lemmas 2 and 3, it follows that $\lim _{t \rightarrow \infty} \Phi(t)=0$. Therefore, taking $t \rightarrow \infty$ in (93) it follows that (84) holds. This completes the proof.

\section{Stationary Distribution}

In this section, we discuss the stationary distribution of model (6) by using Lyapunov function method. We firstly define the diffusion matrix $A(x)=h(x) h^{T}(x)$, where $x=(S, I, R)$,

$$
\begin{aligned}
h(x) & =\left(\begin{array}{llll}
h_{11}(x) & h_{12}(x) & \cdots & h_{1 l}(x) \\
h_{21}(x) & h_{22}(x) & \cdots & h_{2 l}(x) \\
h_{31}(x) & h_{32}(x) & \cdots & h_{3 l}(x)
\end{array}\right), \\
h_{1 j}(x) & =-f(S) g(I) \sigma_{0 j}-f(S) \sigma_{1 j}, \\
h_{2 j}(x) & =f(S) g(I) \sigma_{0 j}-I \sigma_{2 j}, \\
h_{3 j}(x) & =-R \sigma_{3 j} .
\end{aligned}
$$

Furthermore, we denote by $a_{i i}(x)(i=1,2,3)$ the diagonal elements of matrix $A(x)$. We have $a_{i i}(x)=\sum_{j=1}^{l} h_{i j}^{2}(x)$.

Theorem 16. Assume that $\left(\mathbf{H}_{1}\right)$ holds, $f(S)=S$, and there is a constant $\rho>0$ such that $a_{i i}(x)>\rho$, for any $x \in R_{+}^{3}$ and $i=1,2,3, \gamma>p, d_{I}>d_{S}$, and $\gamma\left(d_{S}+d_{R}\right)>p\left(d_{I}+d_{R}\right)$. If $R_{0}>1$ and

$$
\begin{aligned}
& {\left[\frac{\left(d_{S}+p\right)\left[\gamma\left(d_{S}+d_{R}\right)-p\left(d_{I}+d_{R}\right)\right]}{(\gamma-p) \varepsilon}+d_{S}-C_{1}\right] S^{* 2} \wedge\left[\frac{\left(d_{I}+\gamma\right)\left[\gamma\left(d_{S}+d_{R}\right)-p\left(d_{I}+d_{R}\right)\right]}{(\gamma-p) \varepsilon}+d_{I}-C_{2}\right] I^{* 2}} \\
& \wedge\left[\frac{\left(d_{I}-d_{S}\right)\left(d_{R}+\varepsilon\right)}{\gamma-p}+d_{R}-C_{3}\right] R^{* 2}>\frac{\left[\left(d_{S}+d_{I}\right)(\gamma-p) \varepsilon+\left(d_{S}+p+d_{I}+\gamma\right)\left(\gamma\left(d_{S}+d_{R}\right)-p\left(d_{I}+d_{R}\right)\right)\right] I^{*} \sigma_{2}^{2}}{\varepsilon \beta g\left(I^{*}\right)(\gamma-p)} \\
& \quad+C_{1} S^{* 2}+C_{2} I^{* 2}+C_{3} R^{* 2},
\end{aligned}
$$

where

$$
\begin{aligned}
C_{1} & =\frac{2\left[\left(d_{S}+d_{I}\right)(\gamma-p) \varepsilon+\left(d_{S}+p+d_{I}+\gamma\right)\left(\gamma\left(d_{S}+d_{R}\right)-p\left(d_{I}+d_{R}\right)\right)\right] I^{*}\left(g^{\prime}(0)\right)^{2} \sigma_{0}^{2}}{\varepsilon \beta g\left(I^{*}\right)(\gamma-p)} \\
& +\frac{2\left[\gamma\left(d_{S}+d_{R}\right)-p\left(d_{I}+d_{R}\right)\right] \sigma_{1}^{2}}{(\gamma-p) \varepsilon}+\sigma^{2}, \\
C_{2} & =\frac{2\left[\gamma\left(d_{S}+d_{R}\right)-p\left(d_{I}+d_{R}\right)\right] \sigma_{2}^{2}}{(\gamma-p) \varepsilon}+\sigma^{2}, \\
C_{3} & =\frac{\left(d_{I}-d_{S}\right) \sigma_{3}^{2}}{\gamma-p}+\sigma^{2}
\end{aligned}
$$

and $\left(S^{*}, I^{*}, R^{*}\right)$ is the unique endemic equilibrium of model (2), then model (6) has a unique stationary distribution.
Proof. We here use the Lyapunov function method to prove this theorem. The proof given here is similar to Theorem 5.1 
in [11]. But, due to nonlinear function $g(I)$, the Lyapunov function structured in the following is different from that given in [11].

By Lemma 7, it suffices to find a nonnegative Lyapunov function $V(x)$ and compact set $K \subset R_{+}^{3}$ such that $L V(x) \leq-C$ for some $C>0$ and $x \in R_{+}^{3} / K$.

Denote $x=(S, I, R) \in R_{+}^{3}$. Define the function

$$
V_{1}(x)=\frac{1}{2}\left(R-R^{*}\right)^{2} .
$$

Calculating $L V_{1}(x)$, we have

$$
\begin{aligned}
& L V_{1}(x)=\left(R-R^{*}\right) \\
& \quad \cdot\left(p\left(S-S^{*}\right)+\gamma\left(I-I^{*}\right)-\left(d_{R}+\varepsilon\right)\left(R-R^{*}\right)\right) \\
& \quad+\frac{1}{2} \sigma_{3}^{2} R^{2} \leq-\left(d_{R}+\varepsilon-\sigma_{3}^{2}\right)\left(R-R^{*}\right)^{2} \\
& \quad+p\left(S-S^{*}\right)\left(R-R^{*}\right)+\gamma\left(I-I^{*}\right)\left(R-R^{*}\right) \\
& \quad+\sigma_{3}^{2} R^{* 2} .
\end{aligned}
$$

Define the function

$$
V_{2}(x)=I-I^{*}-I^{*} \ln \frac{I}{I^{*}} .
$$

Calculating $L V_{2}(x)$, we have

$$
\begin{aligned}
& L V_{2}(x)=\left(1-\frac{I^{*}}{I}\right)\left(\beta S g(I)-\left(d_{I}+\gamma\right) I\right)+\frac{I^{*}}{2} \\
& \cdot \sum_{j=1}^{l}\left(S \frac{g(I)}{I} \sigma_{0 j}-\sigma_{2 j}\right)^{2}=\left(I-I^{*}\right) \\
& \cdot\left(\beta S\left(\frac{g(I)}{I}-\frac{g\left(I^{*}\right)}{I^{*}}\right)+\beta \frac{g\left(I^{*}\right)}{I^{*}}\left(S-S^{*}\right)\right) \\
& +\frac{1}{2} I^{*} \sigma_{2}^{2}+\frac{1}{2} I^{*} \sigma_{0}^{2} S^{2} \frac{g^{2}(I)}{I^{2}}-\sum_{j=1}^{l} I^{*} S \frac{g(I)}{I} \sigma_{0 j} \sigma_{2 j} \\
& \leq \beta \frac{g\left(I^{*}\right)}{I^{*}}\left(S-S^{*}\right)\left(I-I^{*}\right)+\frac{1}{2} I^{*} \sigma_{2}^{2}+\frac{1}{2} \\
& \cdot I^{*} \sigma_{0}^{2}\left(g^{\prime}(0)\right)^{2} S^{2}-\sum_{j=1}^{l} I^{*} S \frac{g(I)}{I} \sigma_{0 j} \sigma_{2 j} \leq \beta \\
& . \frac{g\left(I^{*}\right)}{I^{*}}\left(S-S^{*}\right)\left(I-I^{*}\right)+2 I^{*} \sigma_{0}^{2}\left(g^{\prime}(0)\right)^{2} \\
& \cdot\left(S-S^{*}\right)^{2}+2 I^{*} \sigma_{0}^{2}\left(g^{\prime}(0)\right)^{2} S^{* 2}+I^{*} \sigma_{2}^{2} .
\end{aligned}
$$

Define the function

$$
V_{3}(x)=\frac{1}{2}\left(S+I-S^{*}-I^{*}\right)^{2} .
$$

Calculating $L V_{3}(x)$, we get

$$
\begin{aligned}
& L V_{3}(x)=\left(S+I-S^{*}-I^{*}\right)\left(-\left(d_{S}+p\right)\left(S-S^{*}\right)\right. \\
& \left.+\varepsilon\left(R-R^{*}\right)-\left(d_{I}+\gamma\right)\left(I-I^{*}\right)\right)+\frac{1}{2} \sigma_{1}^{2} S^{2}+\frac{1}{2} \\
& \cdot \sigma_{2}^{2} I^{2}+\sum_{j=1}^{l} S I \sigma_{1 j} \sigma_{2 j} \leq-\left(\left(d_{S}+p\right)-2 \sigma_{1}^{2}\right)(S \\
& \left.-S^{*}\right)^{2}-\left(d_{I}+\gamma-2 \sigma_{2}^{2}\right)\left(I-I^{*}\right)^{2}+\varepsilon\left(S-S^{*}\right)(R \\
& \left.-R^{*}\right)-\left(d_{S}+p+d_{I}+\gamma\right)\left(S-S^{*}\right)\left(I-I^{*}\right)+\varepsilon(I \\
& \left.-I^{*}\right)\left(R-R^{*}\right)+2 \sigma_{1}^{2} S^{* 2}+2 \sigma_{2}^{2} I^{* 2} .
\end{aligned}
$$

Define the function

$$
V_{4}(x)=\frac{1}{2}\left(S+I+R-S^{*}-I^{*}-R^{*}\right)^{2} .
$$

Calculating $L V_{4}(x)$, we get

$$
\begin{aligned}
& L V_{4}(x)=\left(S+I+R-S^{*}-I^{*}-R^{*}\right) \\
& \cdot\left(\Lambda-d_{S} S-d_{I} I-d_{R} R\right)+\frac{1}{2} \\
& \cdot \sum_{j=1}^{l}\left(S \sigma_{1 j}+I \sigma_{2 j}+R \sigma_{3 j}\right)^{2} \leq-d_{S}\left(S-S^{*}\right)^{2} \\
& -d_{I}\left(I-I^{*}\right)^{2}-d_{R}\left(R-R^{*}\right)^{2}-\left(d_{S}+d_{I}\right) \\
& \cdot\left(S-S^{*}\right)\left(I-I^{*}\right)-\left(d_{S}+d_{R}\right)\left(S-S^{*}\right)\left(R-R^{*}\right) \\
& -\left(d_{I}+d_{R}\right)\left(I-I^{*}\right)\left(R-R^{*}\right)+\frac{1}{2}\left(\sigma_{1}^{2}+\sigma_{2}^{2}+\sigma_{3}^{2}\right) \\
& \cdot\left(S^{2}+I^{2}+R^{2}\right) \leq-\left(d_{S}-\sigma^{2}\right)\left(S-S^{*}\right)^{2} \\
& -\left(d_{I}-\sigma^{2}\right)\left(I-I^{*}\right)^{2}-\left(d_{R}-\sigma^{2}\right)\left(R-R^{*}\right)^{2} \\
& -\left(d_{S}+d_{I}\right)\left(S-S^{*}\right)\left(I-I^{*}\right)-\left(d_{S}+d_{R}\right)\left(S-S^{*}\right) \\
& \cdot\left(R-R^{*}\right)-\left(d_{I}+d_{R}\right)\left(I-I^{*}\right)\left(R-R^{*}\right) \\
& +\sigma^{2}\left(S^{* 2}+I^{* 2}+R^{* 2}\right) .
\end{aligned}
$$

Define the Lyapunov function for model (6) as follows:

$$
\begin{aligned}
V(x)= & \frac{\left[\left(d_{S}+d_{I}\right)(\gamma-p) \varepsilon+\left(d_{S}+p+d_{I}+\gamma\right)\left(\gamma\left(d_{S}+d_{R}\right)-p\left(d_{I}+d_{R}\right)\right)\right] I^{*}}{\varepsilon \beta g\left(I^{*}\right)(\gamma-p)} V_{2}(x)+\frac{d_{I}-d_{S}}{\gamma-p} V_{1}(x) \\
& +\frac{\gamma\left(d_{S}+d_{R}\right)-p\left(d_{I}+d_{R}\right)}{(\gamma-p) \varepsilon} V_{3}(x)+V_{4}(x) .
\end{aligned}
$$


Then from (99), (101), (103), and (105) we have

$$
\begin{aligned}
L V(x) \leq & -\left[\frac{\left(d_{S}+p\right)\left[\gamma\left(d_{S}+d_{R}\right)-p\left(d_{I}+d_{R}\right)\right]}{(\gamma-p) \varepsilon}+d_{S}-C_{1}\right]\left(S-S^{*}\right)^{2} \\
& -\left[\frac{\left(d_{I}+\gamma\right)\left[\gamma\left(d_{S}+d_{R}\right)-p\left(d_{I}+d_{R}\right)\right]}{(\gamma-p) \varepsilon}+d_{I}-C_{2}\right]\left(I-I^{*}\right)^{2}-\left[\frac{\left(d_{I}-d_{S}\right)\left(d_{R}+\varepsilon\right)}{\gamma-p}+d_{R}-C_{3}\right]\left(R-R^{*}\right)^{2} \\
& +C_{1} S^{* 2}+C_{2} I^{* 2}+C_{3} R^{* 2}+\frac{\left[\left(d_{S}+d_{I}\right)(\gamma-p) \varepsilon+\left(d_{S}+p+d_{I}+\gamma\right)\left(\gamma\left(d_{S}+d_{R}\right)-p\left(d_{I}+d_{R}\right)\right)\right] I^{*} \sigma_{2}^{2}}{\varepsilon \beta g\left(I^{*}\right)(\gamma-p)} .
\end{aligned}
$$

If condition (96) holds, then the surface

$$
\begin{aligned}
& {\left[\frac{\left(d_{S}+p\right)\left[\gamma\left(d_{S}+d_{R}\right)-p\left(d_{I}+d_{R}\right)\right]}{(\gamma-p) \varepsilon}+d_{S}-C_{1}\right]\left(S-S^{*}\right)^{2}} \\
& \quad+\left[\frac{\left(d_{I}+\gamma\right)\left[\gamma\left(d_{S}+d_{R}\right)-p\left(d_{I}+d_{R}\right)\right]}{(\gamma-p) \varepsilon}+d_{I}-C_{2}\right]\left(I-I^{*}\right)^{2}+\left[\frac{\left(d_{I}-d_{S}\right)\left(d_{R}+\varepsilon\right)}{\gamma-p}+d_{R}-C_{3}\right]\left(R-R^{*}\right)^{2} \\
& \quad=\frac{\left[\left(d_{S}+d_{I}\right)(\gamma-p) \varepsilon+\left(d_{S}+p+d_{I}+\gamma\right)\left(\gamma\left(d_{S}+d_{R}\right)-p\left(d_{I}+d_{R}\right)\right)\right] I^{*} \sigma_{2}^{2}}{\varepsilon \beta g\left(I^{*}\right)(\gamma-p)}+C_{1} S^{* 2}+C_{2} I^{* 2}+C_{3} R^{* 2}
\end{aligned}
$$

lies in the interior of $R_{+}^{3}$. Hence, we can easily obtain that there exists a constant $C>0$ and a compact set $K$ of $R_{+}^{3}$ such that, for any $x \in R_{+}^{3} / K$,

$$
L V(x) \leq-C
$$

Therefore, model (6) has a unique stationary distribution. This completes the proof.

Remark 17. In fact, the variances of errors usually should be small enough to justify their validity of real data; otherwise, the data may not be considered as a good one. It is clear that when $\sigma_{i j}$ are very small, condition (96) is always satisfied.

\section{Numerical Examples}

To verify the theoretical results in this paper, we next give numerical simulations of model (6).

Throughout the following numerical simulations, we choose $l=2$ and $g(I)=I /\left(1+\omega I^{2}\right)$, where $\omega$ is a positive constant. It is easy to verify that assumption $\left(H_{1}\right)$ holds. By Milstein's higher-order method [29, 30], we drive the corresponding discretization equations of model (6):

$$
\begin{aligned}
S_{i+1} & =S_{i} \\
& +\left((1-q) \Lambda-\frac{\beta f\left(S_{i}\right) I_{i}}{1+\omega I_{i}^{2}}-\left(d_{S_{i}}+P\right) S_{i}+\varepsilon R_{i}\right) \Delta t
\end{aligned}
$$

$$
\begin{aligned}
& -\frac{f\left(S_{i}\right) I_{i}}{1+\omega I_{i}^{2}} \sum_{j=1}^{l}\left(\sigma_{0 j} \xi_{j i} \sqrt{\Delta t}+\frac{1}{2} \sigma_{0 j}^{2}\left(\xi_{j i}^{2}-1\right) \Delta t\right) \\
& -S_{i} \sum_{j=1}^{l}\left(\sigma_{1 j} \xi_{j i} \sqrt{\Delta t}+\frac{1}{2} \sigma_{1 j}^{2}\left(\xi_{j i}^{2}-1\right) \Delta t\right), \\
& I_{i+1}=I_{i}+\left(\frac{\beta f\left(S_{i}\right) I_{i}}{1+\omega I_{i}^{2}}-\left(d_{I}+\gamma\right) I_{i}\right) \Delta t+\frac{f\left(S_{i}\right) I_{i}}{1+\omega I_{i}^{2}} \\
& \cdot \sum_{j=1}^{l}\left(\sigma_{0 j} \xi_{j i} \sqrt{\Delta t}+\frac{1}{2} \sigma_{0 j}^{2}\left(\xi_{j i}^{2}-1\right) \Delta t\right) \\
& -I_{i} \sum_{j=1}^{l}\left(\sigma_{2 j} \xi_{j i} \sqrt{\Delta t}+\frac{1}{2} \sigma_{2 j}^{2}\left(\xi_{j i}^{2}-1\right) \Delta t\right), \\
& R_{i+1}=R_{i}+\left(q \Lambda+p S_{i}+\gamma I_{i}-\left(d_{R}+\varepsilon\right) R_{i}\right) \Delta t \\
& -R_{i} \sum_{j=1}^{l}\left(\sigma_{3 j} \xi_{j i} \sqrt{\Delta t}+\frac{1}{2} \sigma_{3 j}^{2}\left(\xi_{j i}^{2}-1\right) \Delta t\right) .
\end{aligned}
$$

Here, $\xi_{j i}(i=1,2, \ldots, j=1, \ldots, l)$ are $N(0,1)$-distributed independent Gaussian random variables and $\Delta t>0$ is time increment.

Example 1. In model (6), we take $f(S)=S /(1+0.2 S), \Lambda=$ $1.85, q=0.52, \beta=0.52, p=0.24, \varepsilon=0.2, \gamma=0.3$, 


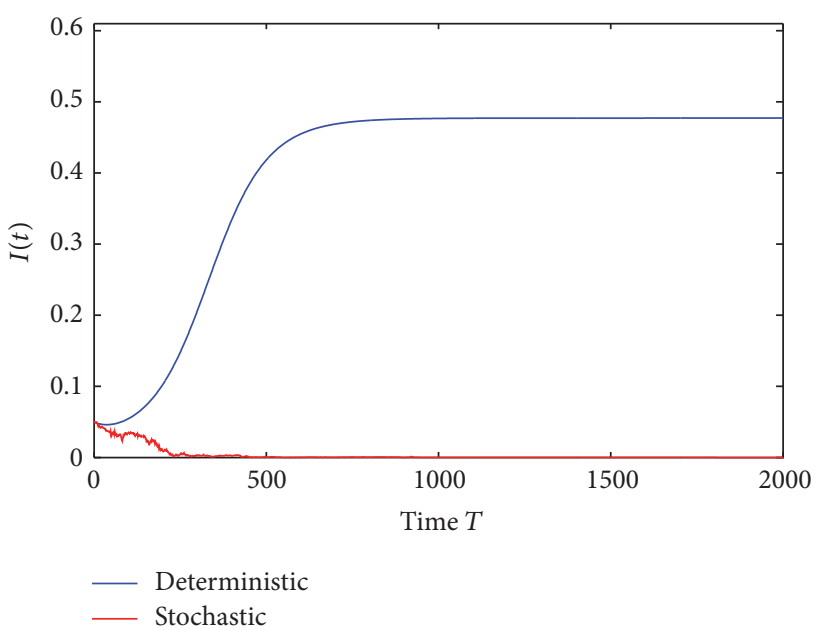

(a)

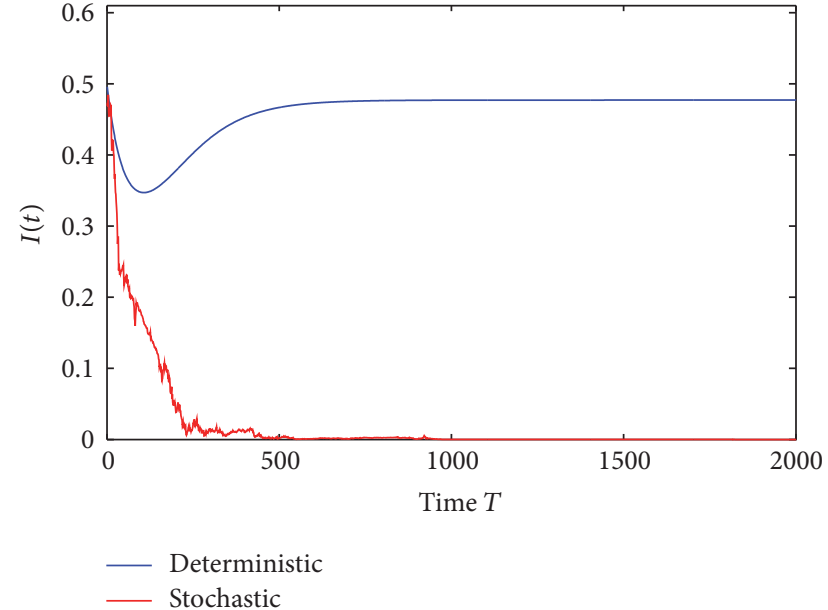

(b)

Figure 1: The path of $I(t)$ for the stochastic model (6) with parameters in Example 1, compared to the corresponding deterministic model. (a) is trajectories of the solution $I(t)$ with the initial value $I(0)=0.05$ and (b) with the initial value $I(0)=0.5$. The disease of model $(6)$ is extinct with probability one.

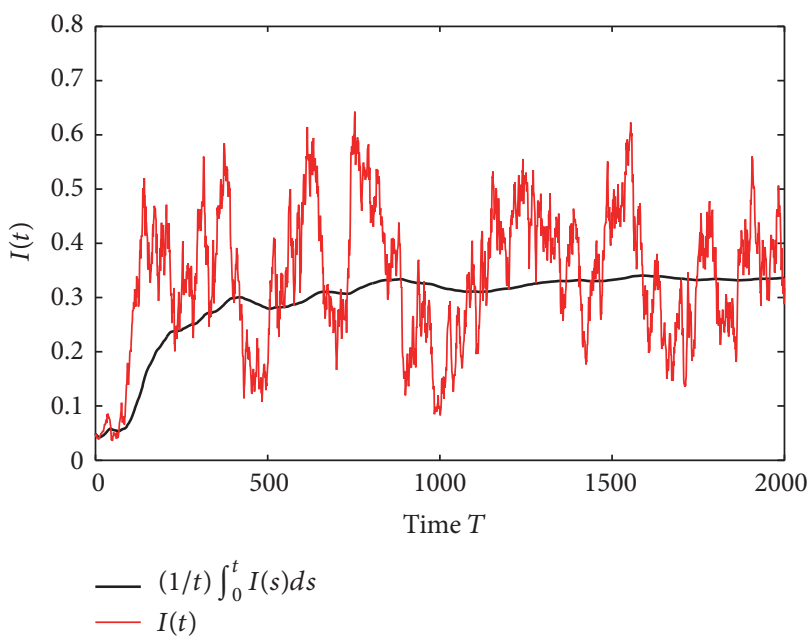

(a)

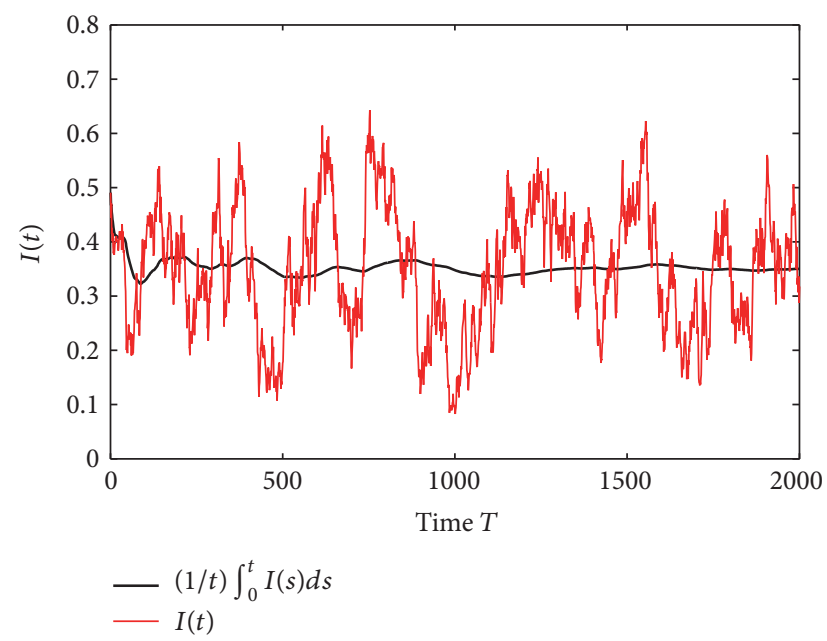

(b)

Figure 2: The paths of $I(t)$ and $(1 / t) \int_{0}^{t} I(s) d s$ for the stochastic model (6) with parameters in Example 3, (a) with the initial value $I(0)=0.05$ and (b) with the initial value $I(0)=0.5$.

$\omega=2, d_{S}=0.4, d_{I}=0.21, d_{R}=0.3, \sigma_{01}=0.15, \sigma_{02}=0.99$ $\sigma_{11}=0.23, \sigma_{12}=0.17, \sigma_{21}=0.14, \sigma_{22}=0.72, \sigma_{31}=0.47$, and $\sigma_{32}=0.93$. By computing, we obtain $\widetilde{R}_{0}=0.8939<$ $1, \sigma_{0}^{2} f\left(S_{0}\right) g^{\prime}(0)-\left(\beta+\sum_{j=1}^{2} \sigma_{0 j} \sigma_{2 j}\right)=0.3442>0$, and $\left(\beta+\sum_{j=1}^{2} \sigma_{0 j} \sigma_{2 j}\right)^{2} / 2 \sigma_{0}^{2}-\left(d_{I}+\gamma+(1 / 2) \sigma_{2}^{2}\right)=0.005>0$. This shows that conditions (a) and (b) of Theorem 9 do not hold. The numerical simulations (see Figure 1) suggest that disease $I(t)$ of model (6) is still stochastically extinct with probability one. Therefore, as an improvement of Theorem 9, we have the following interesting conjecture.
Conjecture 2. Assume $\left(H_{1}\right)$ holds. The disease $I(t)$ in model (6) is stochastically extinct with probability one only when $\widetilde{R}_{0}<$ 1 holds.

Example 3. In model (6), we take $f(S)=S /(1+1.5 S), \Lambda=3$, $q=0.2, \beta=2.1, p=0.3, \varepsilon=0.8, \gamma=0.1, \omega=2$, $d_{S}=0.5, d_{I}=0.8, d_{R}=0.4, \sigma_{01}=0.8, \sigma_{02}=1.2, \sigma_{11}=0.3$, $\sigma_{12}=0.75, \sigma_{21}=0.45, \sigma_{22}=0.8, \sigma_{31}=0.8$, and $\sigma_{32}=0.3$. By computing, we obtain $\widetilde{R}_{0}=1.3554>1$. From the numerical simulations given in Figure 2, it is shown that disease $I(t)$ of model (6) is not only stochastically persistent in the mean but 


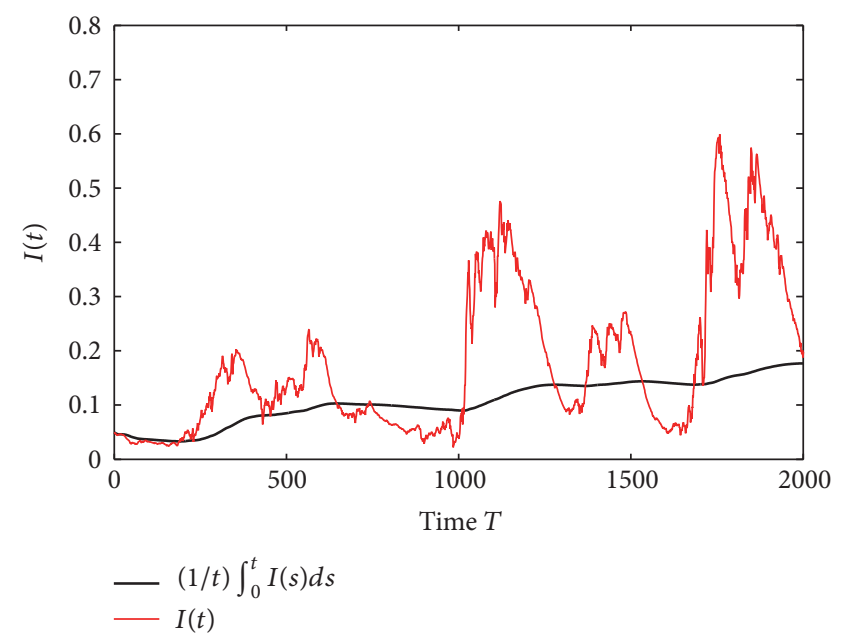

(a)

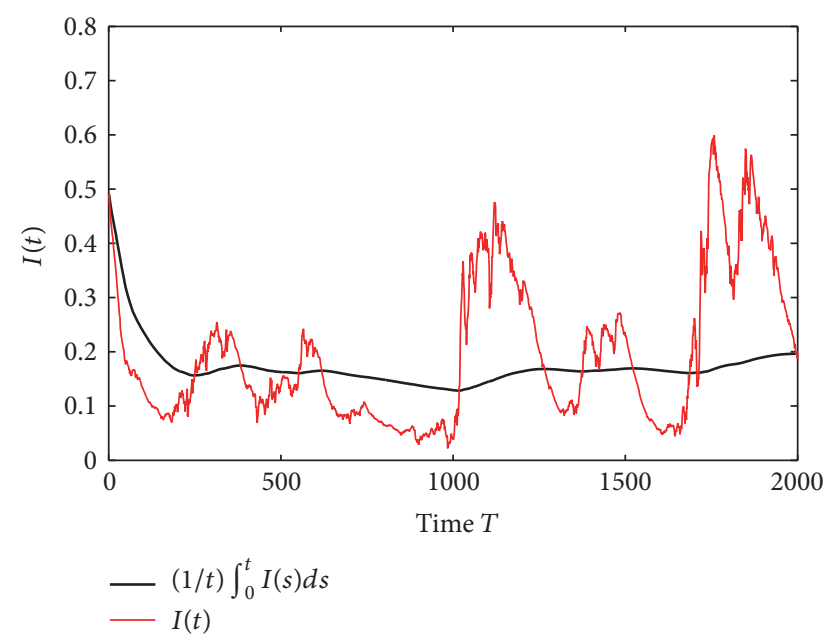

(b)

FIGURE 3: The paths of $I(t)$ and $(1 / t) \int_{0}^{t} I(s) d s$ for the stochastic model (6) with parameters in Example 5, (a) with the initial value $I(0)=0.05$ and (b) with the initial value $I(0)=0.5$.

also stochastically persistent with probability one. Therefore, as an improvement of Theorem 12, we have the following interesting conjecture.

Conjecture 4. Assume $\left(H_{1}\right)$ holds. The disease $I(t)$ in model (6) is stochastically persistent in the mean only when $\widetilde{R}_{0}>1$.

Example 5. In model (6), we take $f(S)=S /(1+0.1 S), \Lambda=1.2$, $q=0.5, \beta=1.5, p=0.9, \varepsilon=1.1, \gamma=0.9, \omega=2, d_{S}=0.6$, $d_{I}=0.35, d_{R}=0.4, \sigma_{01}=0.4, \sigma_{02}=0.2, \sigma_{11}=0.1$, $\sigma_{12}=0.45, \sigma_{21}=0.2, \sigma_{22}=0.1, \sigma_{31}=0.2$, and $\sigma_{32}=0.3$. By computing, we obtain $\bar{R}_{0}=0.8687<1$ and $\widetilde{R}_{0}=1.2931>$ 1 . The numerical simulations given in Figure 3 show that disease $I(t)$ of model (6) is still stochastically permanent in the mean. Therefore, combining Theorem 12 and Theorem 14, we can obtain the following interesting conjecture about the stochastic permanence in the mean of disease $I(t)$.
Conjecture 6. Assume $\left(H_{1}\right)$ holds. The disease $I(t)$ in model (6) is stochastically permanent in the mean only when $\widetilde{R}_{0}>1$.

Example 7. In model (6), we take $f(S)=S, \Lambda=0.67$, $q=0.02, \beta=1.7, p=0.05, \varepsilon=3, \gamma=0.99, \omega=4$, $d_{S}=0.29, d_{I}=0.53, d_{R}=0.39, \sigma_{01}=0.025, \sigma_{02}=0.02$, $\sigma_{11}=0.0121, \sigma_{12}=0.01, \sigma_{21}=0, \sigma_{22}=0, \sigma_{31}=0.02$, and $\sigma_{32}=0.01$. By computing, we obtain that the basic reproduction number for deterministic model (2) is $R_{0}=$ $2.5279>1$ and the unique endemic equilibrium of model (2) is $\left(S^{*}, I^{*}, R^{*}\right)=(1.4230,0.3845,0.1372)$. Furthermore, we can verify that there is a constant $\rho>0$ such that $a_{i i}(x)>\rho$ for any $x \in R_{+}^{3}(i=1,2,3), d_{I}-d_{S}=0.24>0, \gamma-p=0.94>0$, $\gamma\left(d_{S}+d_{R}\right)-p\left(d_{I}+d_{R}\right)=0.6272>0$, and

$$
\begin{aligned}
& {\left[\frac{\left(d_{S}+p\right)\left[\gamma\left(d_{S}+d_{R}\right)-p\left(d_{I}+d_{R}\right)\right]}{(\gamma-p) \varepsilon}+d_{S}-C_{1}\right] S^{* 2} \wedge\left[\frac{\left(d_{I}+\gamma\right)\left[\gamma\left(d_{S}+d_{R}\right)-p\left(d_{I}+d_{R}\right)\right]}{(\gamma-p) \varepsilon}+d_{I}-C_{2}\right] I^{* 2}} \\
& \quad \wedge\left[\frac{\left(d_{I}-d_{S}\right)\left(d_{R}+\varepsilon\right)}{\gamma-p}+d_{R}-C_{3}\right] R^{* 2}-\frac{\left[\left(d_{S}+d_{I}\right)(\gamma-p) \varepsilon+\left(d_{S}+p+d_{I}+\gamma\right)\left(\gamma\left(d_{S}+d_{R}\right)-p\left(d_{I}+d_{R}\right)\right)\right] I^{*} \sigma_{2}^{2}}{\varepsilon \beta g\left(I^{*}\right)(\gamma-p)} \\
& \quad+C_{1} S^{* 2}+C_{2} I^{* 2}+C_{3} R^{* 2}=0.0147>0 .
\end{aligned}
$$

That is, all conditions in Theorem 16 are satisfied. The stationary distributions about the susceptible, infected, and removed individuals obtained through the numerical simulations are reported in Figure 4, which shows that after some initial transients the population densities fluctuate around the deterministic steady-state values $S^{*}=1.4230, I^{*}=0.3845$, and $R^{*}=0.1372$.
Example 8. In model (6), we take $f(S)=S /(1+0.4 S), \Lambda=2.5$, $q=0.5, \beta=1.4, p=0.7, \varepsilon=0.9, \gamma=0.51, \omega=1.89$, $d_{S}=0.7, d_{I}=0.45, d_{R}=0.58, \sigma_{01}=0.4, \sigma_{02}=0.2$, $\sigma_{11}=0.21, \sigma_{12}=0.1, \sigma_{21}=0.1, \sigma_{22}=0.24, \sigma_{31}=$ 0.2 , and $\sigma_{32}=0.1$. By computing, we obtain that the basic reproduction number for deterministic model (2) is $R_{0}=$ $1.6484>1$ and the unique endemic equilibrium of model 

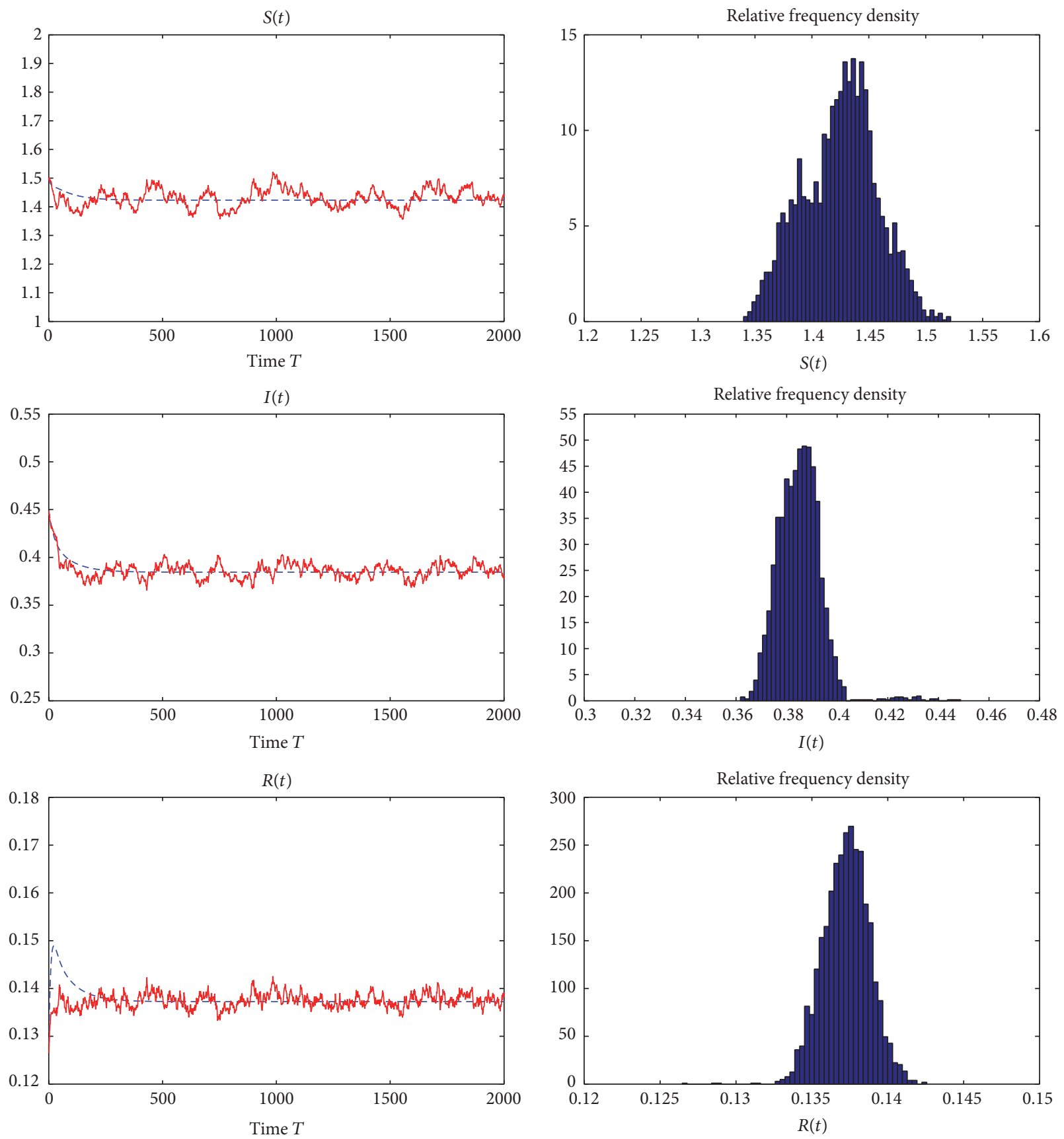

FIgURE 4: The solution of stochastic model (6) and its histogram with parameters in Example 7.

(2) is $\left(S^{*}, I^{*}, R^{*}\right)=(1.7242,0.5082,1.8352)$. Furthermore, we can verify that there is not a constant $\rho>0$ such that $a_{i i}(x)>\rho$ for any $x \in R_{+}^{3}$ and $i=1,2,3, d_{I}-d_{S}=-0.25<0$, $\gamma-p=-0.19<0, \gamma\left(d_{S}+d_{R}\right)-p\left(d_{I}+d_{R}\right)=-0.0682<0$ and

$$
\begin{aligned}
& {\left[\frac{\left(d_{S}+p\right)\left[\gamma\left(d_{S}+d_{R}\right)-p\left(d_{I}+d_{R}\right)\right]}{(\gamma-p) \varepsilon}+d_{S}-C_{1}\right] S^{* 2} \wedge\left[\frac{\left(d_{I}+\gamma\right)\left[\gamma\left(d_{S}+d_{R}\right)-p\left(d_{I}+d_{R}\right)\right]}{(\gamma-p) \varepsilon}+d_{I}-C_{2}\right] I^{* 2}} \\
& \quad \wedge\left[\frac{\left(d_{I}-d_{S}\right)\left(d_{R}+\varepsilon\right)}{\gamma-p}+d_{R}-C_{3}\right] R^{* 2}-\frac{\left[\left(d_{S}+d_{I}\right)(\gamma-p) \varepsilon+\left(d_{S}+p+d_{I}+\gamma\right)\left(\gamma\left(d_{S}+d_{R}\right)-p\left(d_{I}+d_{R}\right)\right)\right] I^{*} \sigma_{2}^{2}}{\varepsilon \beta g\left(I^{*}\right)(\gamma-p)} \\
& \quad+C_{1} S^{* 2}+C_{2} I^{* 2}+C_{3} R^{* 2}=-5.5051<0 .
\end{aligned}
$$



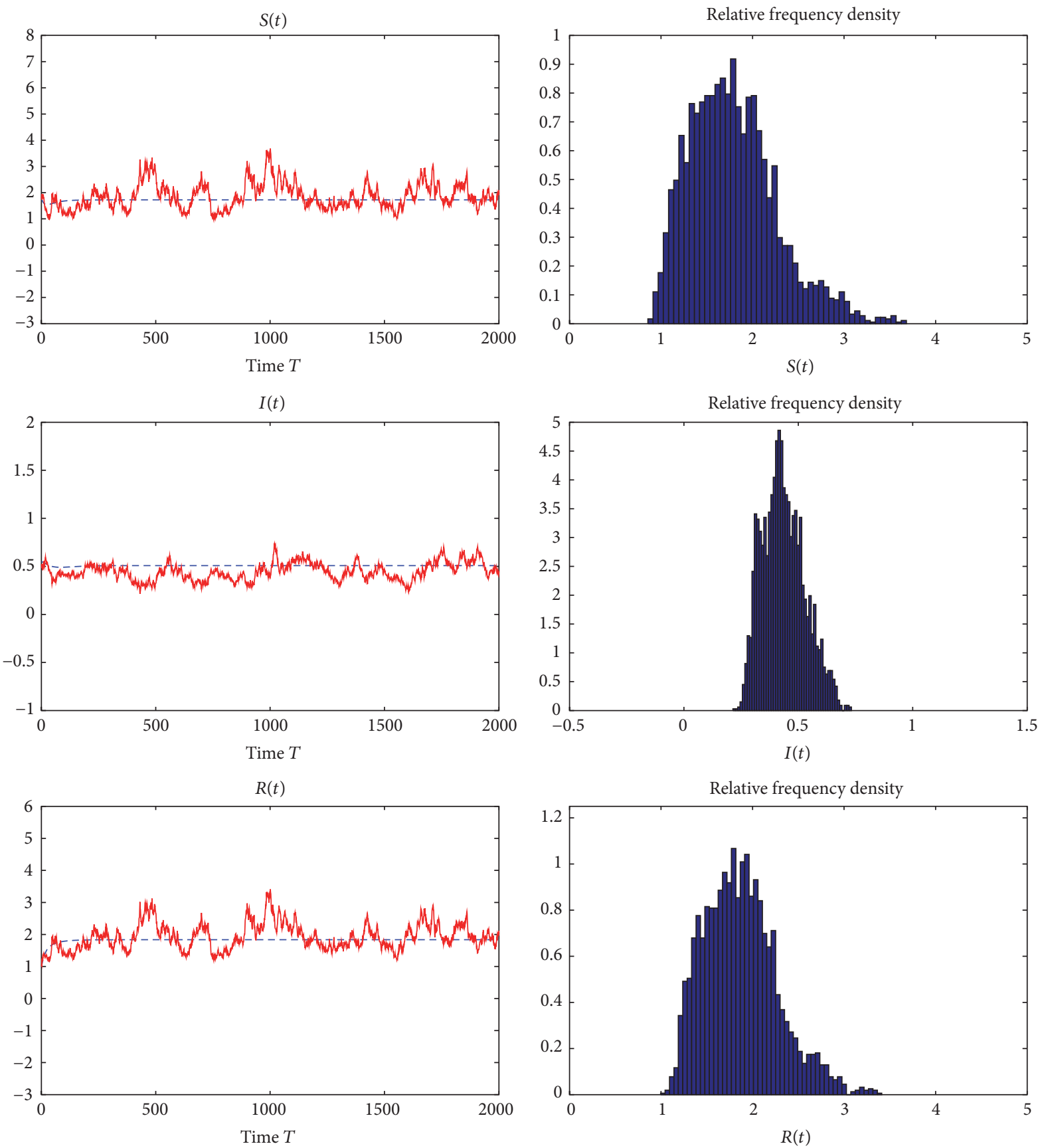

FIGURE 5: The solution of stochastic model (6) and its histogram with parameters in Example 8.

That is, the conditions in Theorem 16 are not satisfied. However, we obtain that threshold value $\widetilde{R}_{0}=2.7192>$ 1. The numerical simulations given in Figure 5 show the stationary distributions about the susceptible, infected, and removed individuals. Therefore, we can obtain the following interesting conjecture about the stationary distribution for model (6), as described in the conclusion part.

Conjecture 9. Assume $\left(H_{1}\right)$ holds. Model (6) has a unique stationary distribution only when $\widetilde{R}_{0}>1$.

\section{Conclusion}

In this paper, as an extension of the results given in [11, 25], we investigated the dynamical behaviors for a stochastic SIRS epidemic model (6) with nonlinear incidence and vaccination. In model (6), the disease transmission coefficient $\beta$ and the removal rates $d_{S}, d_{I}$, and $d_{R}$ are affected by noise. Some new basic properties of model (6) are found in Lemmas 2, 3, and 5. Applying these lemmas, we established a series of new threshold value criteria on the stochastic extinction, 
persistence in the mean, and permanence in the mean of the disease with probability one. Furthermore, by using the Lyapunov function method, a sufficient condition on the existence of unique stationary distribution for model (6) is also obtained.

The stochastic persistence and permanence in the mean of the disease for model (6) are established in this paper only for the special cases: $f(S) \equiv S$ and (1) $\sigma_{0 j}=0(1 \leq j \leq l)$ or (2) $\sigma_{1 j}=\sigma_{2 j}=\sigma_{3 j}=0(1 \leq j \leq l)$. However, for the general model (6), particularly, $f(S) \neq S$ and $\left(\sigma_{1 j}, \sigma_{2 j}, \sigma_{3 j}\right) \neq$ $(0,0,0)(1 \leq j \leq l)$, whether we also can establish similar results still is an interesting open problem.

In fact, under the above case, from the proofs of Theorems 12 and 14, we can see that an important question is to deal with terms $\beta f(S(t))$ and $f^{2}(S(t)) g^{\prime}(I(t))$. If we may get

$$
\begin{aligned}
\beta f(S(t)) \geq & \beta f\left(S_{0}\right)+v_{1}\left(S(t)-S_{0}\right) \text { a.s., } \\
f^{2}(S(t)) g^{\prime}(I(t)) \leq & f^{2}\left(S_{0}\right) g^{\prime}(0) \\
& +v_{2}\left(S(t)-S_{0}\right) \text { a.s., }
\end{aligned}
$$

where $v_{1}$ and $v_{2}$ are two positive constants; then the following perfect result may be established.

Assume that $\left(H_{1}\right)$ holds. If $\widetilde{R}_{0}>1$, then disease $I$ in model (6) is stochastically persistent in the mean; that is,

$$
\liminf _{t \rightarrow \infty} \frac{1}{t} \int_{0}^{t} I(r) d r>0 \text { a.s. }
$$

Another important open problem is about the existence of stationary distribution of model (6), that is, whether we can establish a similar result as in Theorem 16 when $f(S)$ is a nonlinear function. The best perfect result on the stationary distribution is to prove that model (6) possesses a unique stationary distribution only when threshold value $\widetilde{R}_{0}>1$. But this is a very difficult open problem.

However, the numerical examples given in Section 6 propose some affirmative answer for above open problems.

\section{Competing Interests}

The authors declare that they have no competing interests.

\section{Acknowledgments}

This research is supported by the Natural Science Foundation of Xinjiang (Grant no. 2016D03022) and the National Natural Science Foundation of China (Grant nos. 11401512, 11271312, and 11660176).

\section{References}

[1] E. Beretta, V. Kolmanovskii, and L. Shaikhet, "Stability of epidemic model with time delays influenced by stochastic perturbations," Mathematics and Computers in Simulation, vol. 45, no. 3-4, pp. 269-277, 1998.

[2] E. Tornatore, S. M. Buccellato, and P. Vetro, "Stability of a stochastic SIR system," Physica A: Statistical Mechanics and Its Applications, vol. 354, no. 1-4, pp. 111-126, 2005.
[3] N. Dalal, D. Greenhalgh, and X. Mao, "A stochastic model of AIDS and condom use," Journal of Mathematical Analysis and Applications, vol. 325, no. 1, pp. 36-53, 2007.

[4] N. Dalal, D. Greenhalgh, and X. Mao, "A stochastic model for internal HIV dynamics," Journal of Mathematical Analysis and Applications, vol. 341, no. 2, pp. 1084-1101, 2008.

[5] A. Gray, D. Greenhalgh, L. Hu, X. Mao, and J. Pan, "A stochastic differential equation SIS epidemic model," SIAM Journal on Applied Mathematics, vol. 71, no. 3, pp. 876-902, 2011.

[6] A. Gray, D. Greenhalgh, X. Mao, and J. Pan, "The SIS epidemic model with Markovian switching," Journal of Mathematical Analysis and Applications, vol. 394, no. 2, pp. 496-516, 2012.

[7] A. Lahrouz and A. Settati, "Necessary and sufficient condition for extinction and persistence of SIRS system with random perturbation," Applied Mathematics and Computation, vol. 233, pp. 10-19, 2014.

[8] D. Jiang, C. Ji, N. Shi, and J. Yu, "The long time behavior of DI SIR epidemic model with stochastic perturbation," Journal of Mathematical Analysis and Applications, vol. 372, no. 1, pp. 162-180, 2010.

[9] M. Liu, C. Bai, and K. Wang, "Asymptotic stability of a twogroup stochastic SEIR model with infinite delays," Communications in Nonlinear Science and Numerical Simulation, vol. 19, no. 10, pp. 3444-3453, 2014.

[10] Q. Lu, "Stability of SIRS system with random perturbations," Physica A: Statistical Mechanics and Its Applications, vol. 388, no. 18, pp. 3677-3686, 2009.

[11] Q. Yang and X. Mao, "Stochastic dynamics of SIRS epidemic models with random perturbation," Mathematical Biosciences and Engineering, vol. 11, no. 4, pp. 1003-1025, 2014.

[12] D. Zhao, T. Zhang, and S. Yuan, "The threshold of a stochastic SIVS epidemic model with nonlinear saturated incidence," Physica A. Statistical Mechanics and its Applications, vol. 443, pp. 372-379, 2016.

[13] Y. Zhao, D. Jiang, and D. O'Regan, “The extinction and persistence of the stochastic SIS epidemic model with vaccination," Physica A, vol. 392, no. 20, pp. 4916-4927, 2013.

[14] Y. Zhao and D. Jiang, "The threshold of a stochastic SIS epidemic model with vaccination," Applied Mathematics and Computation, vol. 243, pp. 718-727, 2014.

[15] C. Ji and D. Jiang, "Threshold behaviour of a stochastic SIR model," Applied Mathematical Modelling, vol. 38, no. 21-22, pp. 5067-5079, 2014.

[16] Q. Yang and X. Mao, "Extinction and recurrence of multigroup SEIR epidemic models with stochastic perturbations," Nonlinear Analysis: Real World Applications, vol. 14, no. 3, pp. 1434-1456, 2013.

[17] P. J. Witbooi, "Stability of an SEIR epidemic model with independent stochastic perturbations," Physica A, vol. 392, no. 20, pp. 4928-4936, 2013.

[18] A. Lahrouz and A. Settati, "Qualitative study of a nonlinear stochastic SIRS epidemic system," Stochastic Analysis and Applications, vol. 32, no. 6, pp. 992-1008, 2014.

[19] Q. Yang, D. Jiang, N. Shi, and C. Ji, "The ergodicity and extinction of stochastically perturbed SIR and SEIR epidemic models with saturated incidence," Journal of Mathematical Analysis and Applications, vol. 388, no. 1, pp. 248-271, 2012.

[20] Y. Zhao and D. Jiang, "The threshold of a stochastic SIRS epidemic model with saturated incidence," Applied Mathematics Letters, vol. 34, pp. 90-93, 2014. 
[21] Y. Zhou, W. Zhang, S. Yuan, and H. Hu, "Persistence and extinction in stochastic SIRS models with general nonlinear incidence rate," Electronic Journal of Differential Equations, vol. 2014, article 42, pp. 1-17, 2014.

[22] H. Liu, Q. Yang, and D. Jiang, "The asymptotic behavior of stochastically perturbed DI SIR epidemic models with saturated incidences," Automatica, vol. 48, no. 5, pp. 820-825, 2012.

[23] A. Lahrouz and L. Omari, "Extinction and stationary distribution of a stochastic SIRS epidemic model with non-linear incidence," Statistics \& Probability Letters, vol. 83, no. 4, pp. 960968, 2013.

[24] Q. Liu and Q. Chen, "Analysis of the deterministic and stochastic SIRS epidemic models with nonlinear incidence," Physica A: Statistical Mechanics and Its Applications, vol. 428, pp. 140-153, 2015.

[25] T. Tang, Z. Teng, and Z. Li, "Threshold behavior in a class of stochastic SIRS epidemic models with nonlinear incidence," Stochastic Analysis and Applications, vol. 33, no. 6, pp. 994-1019, 2015.

[26] A. Lahrouz, L. Omari, D. Kiouach, and A. Belmaâti, "Complete global stability for an SIRS epidemic model with generalized non-linear incidence and vaccination," Applied Mathematics and Computation, vol. 218, no. 11, pp. 6519-6525, 2012.

[27] X. Mao, Stochastic Differential Equations and Applications, Horwood, Chichester, UK, 1997.

[28] R. Z. Hasminskii, Stochastic stability of differential equations, vol. 7 of Monographs and Textbooks on Mechanics of Solids and Fluids: Mechanics and Analysis, Springer-Verlag, Berlin, Germany, 1980.

[29] M. Carletti, K. Burrage, and P. M. Burrage, "Numerical simulation of stochastic ordinary differential equations in biomathematical modelling," Mathematics and Computers in Simulation, vol. 64, no. 2, pp. 271-277, 2004.

[30] D. J. Higham, "An algorithmic introduction to numerical simulation of stochastic differential equations," SIAM Review, vol. 43, no. 3, pp. 525-546, 2001. 


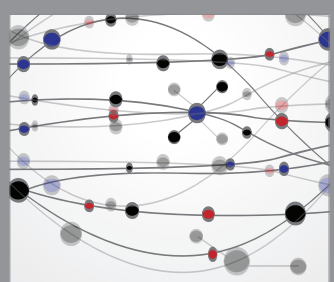

The Scientific World Journal
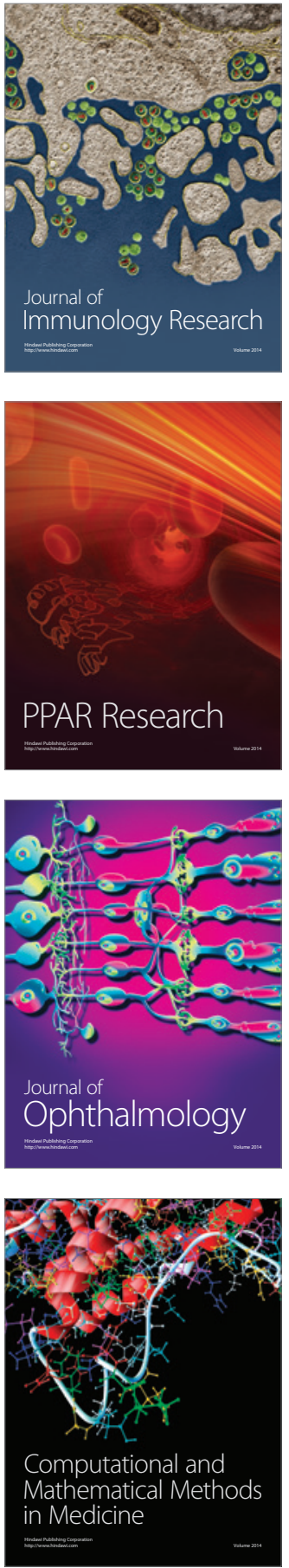

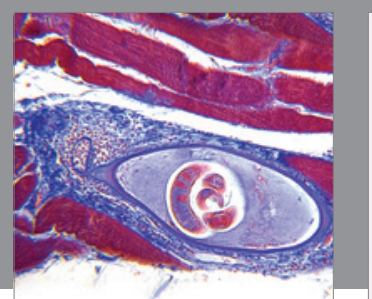

Gastroenterology Research and Practice
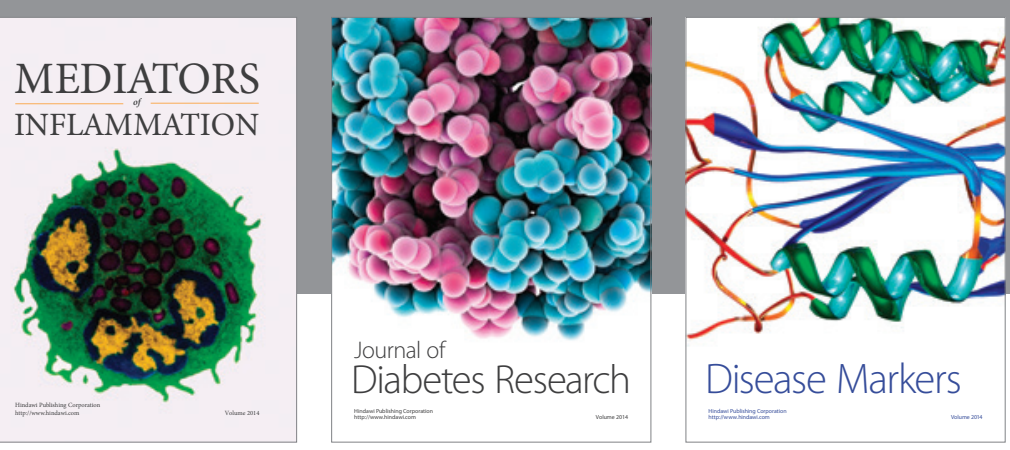

Disease Markers

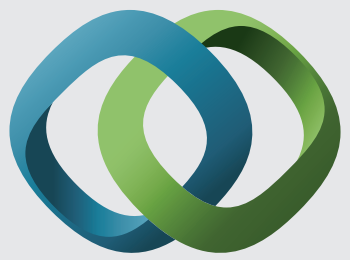

\section{Hindawi}

Submit your manuscripts at

https://www.hindawi.com
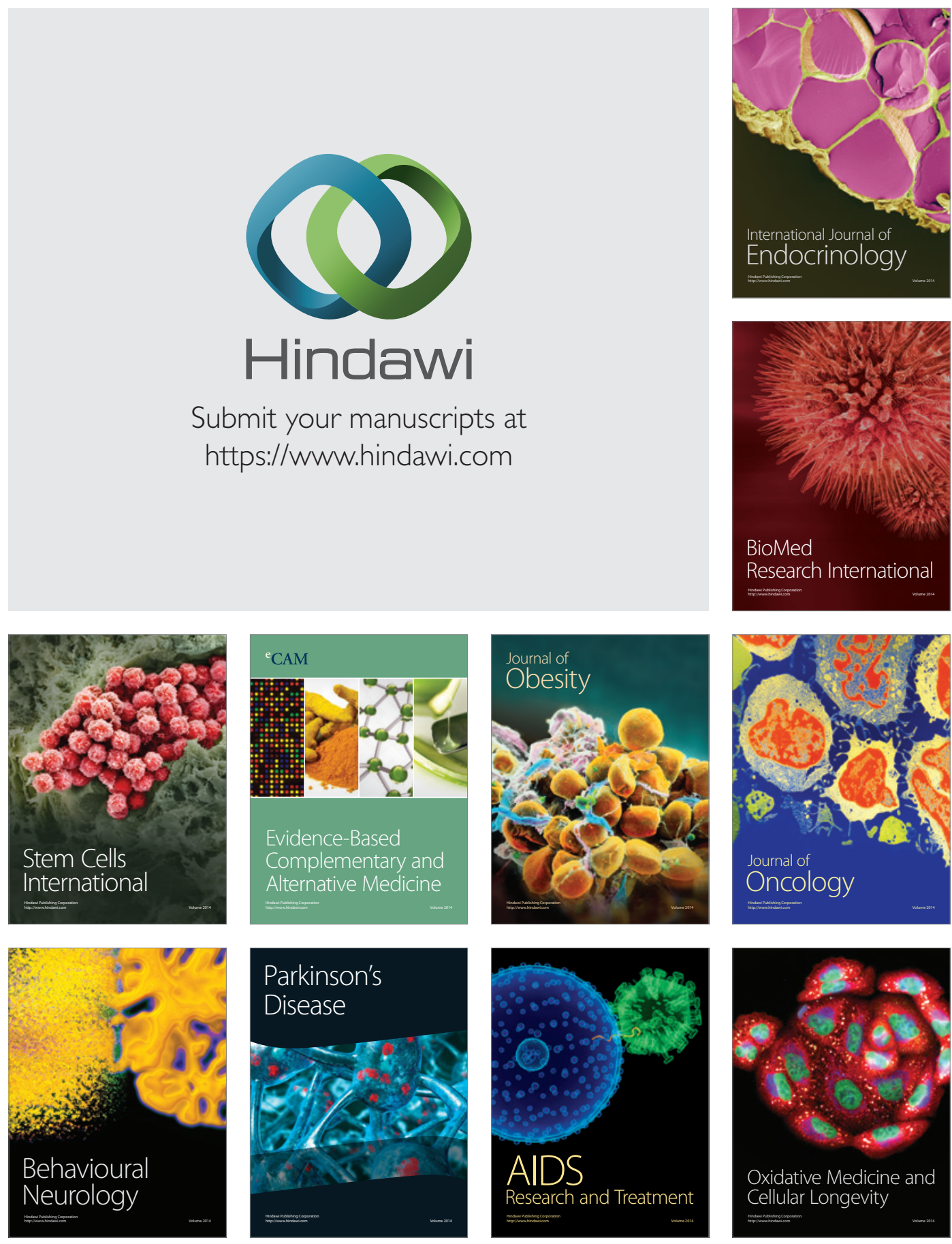